\title{
Hydrodynamic characteristics of flow past a square cylinder at moderate Reynolds numbers
}

HONGYI JIANG ${ }^{1}$, and LIANG CHENG ${ }^{1,2 \dagger}$

\author{
${ }^{1}$ School of Engineering, The University of Western Australia, 35 Stirling Highway, \\ Crawley, WA 6009, Australia \\ ${ }^{2}$ State Key Laboratory of Coastal and Offshore Engineering, Dalian University of \\ Technology, Dalian, 116024, China
}

\begin{abstract}
Two-dimensional (2D) and three-dimensional (3D) hydrodynamic characteristics of flow past a square cylinder are investigated using direct numerical simulations. The variations of the hydrodynamic forces and wake flow characteristics with the Reynolds number $(R e)$ are examined for $R e=10$ to 400 with a high resolution in $R e$. Distinct features in the variation trends of the hydrodynamic forces with $R e$ are examined and explained. In addition, for the 3D flows up to $R e=400$, three critical conditions are observed at $R e$ of approximately 165.7, 210 and 330. At the onset of the secondary wake instability of $R e=165.7$, a sudden increase or decrease of the 3D flow property with respect to its $2 \mathrm{D}$ counterpart is observed, which is due to the sudden increase in the flow three-dimensionality at the mode A instability that is subcritical in nature. The second critical condition is because for $R e>210$ the mode $\mathrm{B}$ flow structure becomes increasingly disordered and the wake flow becomes increasingly turbulent, such that the 3D flow property is increasingly deviated from its 2D counterpart. The third critical condition at $R e \sim 330$ is related to the disappearance and reappearance of the recirculation regions on the upper and lower sides of the cylinder. While the first two critical conditions are also observed for flow past a circular cylinder, the third one is not, since the geometry of a circular cylinder does not give rise to recirculation regions on the upper and lower sides of the cylinder.
\end{abstract}

\footnotetext{
${ }^{\dagger}$ Correspondence author: liang.cheng@uwa.edu.au
} 


\section{Introduction}

Steady incoming flow past a nominally two-dimensional (2D) bluff body has been a classical problem in fluid mechanics owing to its fundamental and practical significance. Typical bluff bodies include circular and square cylinders. The scenario of steady incoming flow past a smooth circular/square cylinder (referred to as "flow past a circular/square cylinder" hereafter) is governed by a single dimensionless parameter, the Reynolds number $\operatorname{Re}(=U D / v)$, which is defined based on the incoming flow velocity $(U)$, the length scale of the cylinder perpendicular to the incoming flow $(D)$, and the kinematic viscosity of the fluid $(v)$. Specifically, for a circular cylinder the length scale is the diameter of the cylinder, while for a square cylinder aligned with sides perpendicular and parallel to the incoming flow the length scale is the side length of the cylinder.

The wake transition regimes of flow past a circular cylinder have been well studied in the literature. With increasing $R e$ a few flow regimes have been discovered (see, e.g., Williamson, 1996a):

(i) Laminar steady regime $(R e<47)$. The flow is steady, with two recirculation regions formed symmetrical about the wake centreline.

(ii) Laminar vortex shedding regime ( $R e \sim 47$ to 190 ). At $R e=47$, the primary wake instability develops as a result of a Hopf bifurcation. Periodic shedding of the Kármán vortices is observed in this regime.

(iii) Three-dimensional (3D) wake transition regime ( $R e \sim 190$ to 270). At $R e \sim$ 190, the secondary wake instability develops (i.e. the flow transitions from $2 \mathrm{D}$ to $3 \mathrm{D}$ ). The relatively large-scale mode A flow structure (with a spanwise wavelength of $\sim 4 D$ and an out-of-phase sequence between the neighbouring streamwise vortices) with vortex dislocations is observed for $R e \sim 190$ to 230, while a gradual wake transition from mode A with dislocations to the finer-scale mode B flow structure (with a spanwise wavelength of $\sim 0.8 D$ and an in-phase sequence between the neighbouring streamwise vortices) takes place at $R e \sim 230$ to 260 . 
(iv) Wake transition to turbulence ( $R e \sim 270$ to 1200$)$. The mode B flow structure becomes increasingly disordered and the wake flow becomes increasingly turbulent. It is noted that the shear layer instability at $R e \gtrsim 1200$ is outside the scope of the present study.

For flow past a circular cylinder, the hydrodynamic forces and wake flow characteristics for each of the wake transition regimes have been quantified by a number of experimental and numerical studies. Through eliminating the "end effects" of the model cylinder that were commonly encountered in early experimental studies (see, e.g., Williamson, 1989), Williamson (1996b) made accurate measurements of the Strouhal-Reynolds number $(S t-R e)$ relationship for $R e$ up to approximately 300 . Previous experimental studies have also reported relationships between $R e$ and a number of flow properties, such as the base pressure coefficient (Williamson and Roshko, 1990; Williamson, 1996a), the formation length $L_{f}$ (Noca et al., 1998), the root-mean-square lift coefficient (Norberg, 2003), the separation angle (Wu et al., 2004), etc. On the other hand, based on direct numerical simulations (DNS), Henderson (1995) and Posdziech and Grundmann (2007) reported various 2D hydrodynamic characteristics for flow past a circular cylinder, while Jiang et al. (2016) and Jiang and Cheng (2017) reported 3D hydrodynamic characteristics. Excellent agreements of the experimental and numerical results have been reached for the $S t-R e$ relationship reported in Williamson (1996b) and Jiang et al. (2016), and for the $L_{f}-R e$ relationship reported in Noca et al. (1998) and Jiang and Cheng (2017).

Similarly, the wake transition regimes of flow past a square cylinder are summarized as follows:

(i) Laminar steady regime $(\mathrm{Re} \lesssim 46)$.

(ii) Laminar vortex shedding regime ( $R e \sim 46$ to 166). The critical $R e$ for the primary wake instability was identified at $47 \pm 2$ in Sohankar et al. (1997), 45 in Saha et al. (2000), 44.7 in Park and Yang (2016), and 46 in Jiang et al. (2018).

(iii) Three-dimensional wake transition regime ( $R e \sim 166$ to 210$)$. The critical $R e$ for the secondary wake instability (i.e. the mode A instability) was identified 
at 164 in Sheard et al. (2009), 166.5 in Choi et al. (2012), 166.6 in Park and Yang (2016), and 165.7 in Jiang et al. (2018). Within the 3D wake transition regime, a gradual wake transition from mode $\mathrm{A}$ with dislocations to mode $\mathrm{B}$ takes place at $R e \sim 185$ to 210 (Jiang et al., 2018).

(iv) Wake transition to turbulence $(R e \gtrsim 210)$.

For flow past a square cylinder, the relationships between $R e$ and the hydrodynamic forces have been reported by only a limited number of studies. Fig. 1 shows the variations of $S t$ and the time-averaged drag coefficient (the two most commonly-studied flow properties) with $R e$ for a few studies available in the literature, together with the DNS results of the present study. In contrast to the aforementioned good agreement of different studies for a circular cylinder, considerable scatters are observed in Fig. 1 for a square cylinder, especially for the range of $R e$ beyond the secondary wake instability, since the 3D flows are more difficult to be measured or simulated accurately. In light of this, the first aim of the present study is to establish a more accurate database of the hydrodynamic forces and wake flow characteristics for flow past a square cylinder. 
(a)

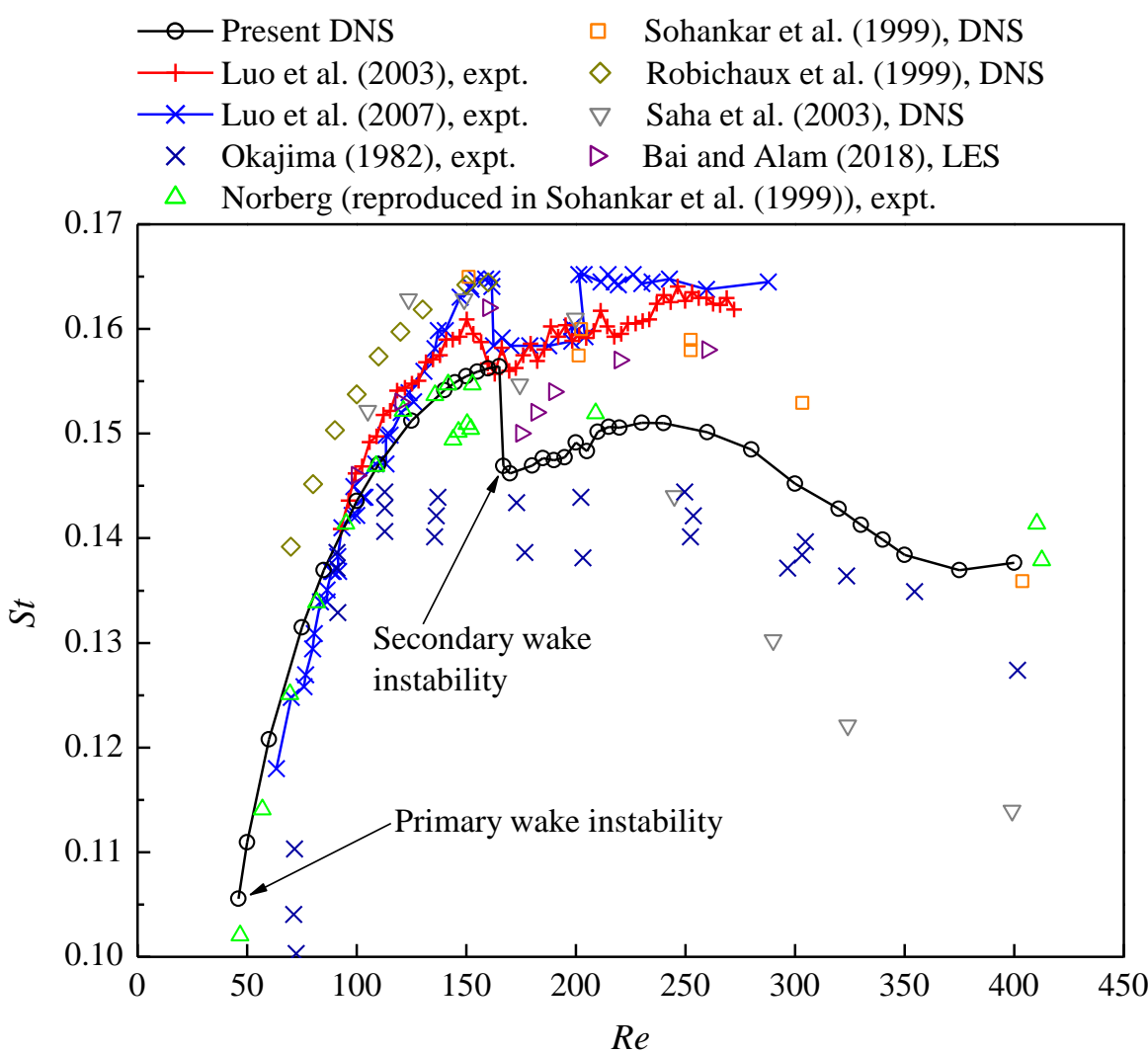

(b)

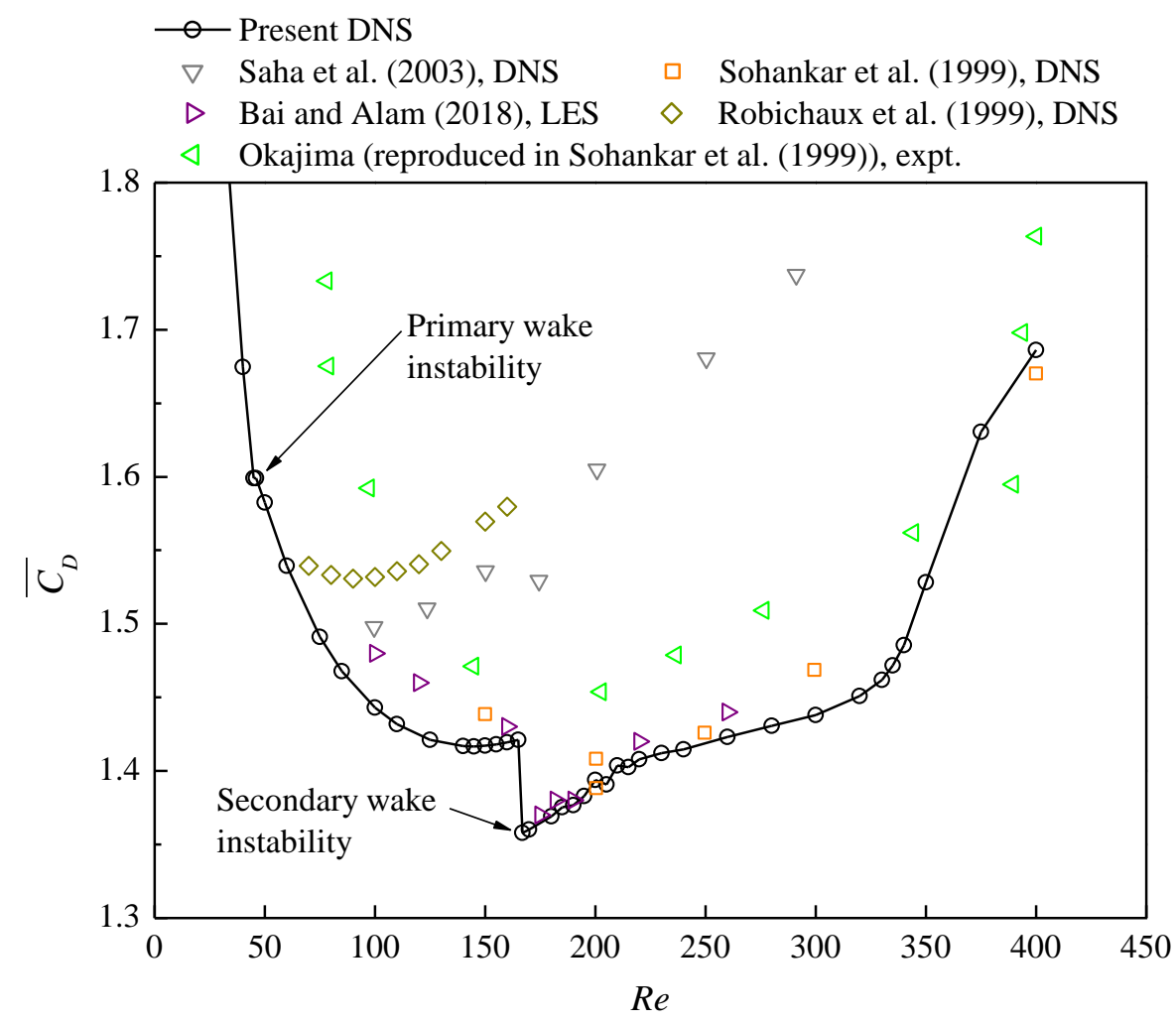

Fig. 1. Variations of (a) the Strouhal number and (b) the time-averaged drag coefficient with $R e$ for a few studies available in the literature. 
In addition, the second aim of the present study is to explore the physical mechanisms responsible for the variations of the hydrodynamic forces and wake flow characteristics with Re. Different variation trends are expected for different wake transition regimes. For example, for flow past a circular cylinder, with increase in $R e$ the formation length increases in the laminar steady regime, followed by a decrease in the laminar vortex shedding regime and an increase beyond the 3D wake transition regime (see, e.g., Williamson, 1996a), while a sudden increase is observed at the onset of the secondary wake instability (Jiang and Cheng, 2017). The critical Re values between the wake transition regimes (i.e. $R e$ of approximately 47, 190 and 270 for the case of a circular cylinder) can be identified by the discontinuities in (i) the relationship between $R e$ and some flow properties (such as the hydrodynamic forces on the cylinder and the flow characteristics in the wake), and (ii) the relationship between $R e$ and the gradient of variation of some flow properties with $R e$. However, such discontinuities between the wake transition regimes for a square cylinder could hardly be identified by some previous results shown in Fig. 1, especially when relatively large intervals of $R e$ were selected. High-quality data over the wake transition regimes would be necessary to shed light on the underlying physical mechanisms responsible for the flow behaviours.

\section{Numerical model}

\subsection{Numerical method}

OpenFOAM (www.openfoam.org) has been adopted in this study in solving the continuity and incompressible Navier-Stokes equations:

$$
\begin{aligned}
& \frac{\partial u_{i}}{\partial x_{i}}=0 \\
& \frac{\partial u_{i}}{\partial t}+u_{j} \frac{\partial u_{i}}{\partial x_{j}}=-\frac{1}{\rho} \frac{\partial p}{\partial x_{i}}+v \frac{\partial^{2} u_{i}}{\partial x_{j} \partial x_{j}}
\end{aligned}
$$

where $\left(x_{1}, x_{2}, x_{3}\right)=(x, y, z)$ are Cartesian coordinates, $u_{i}$ is the velocity component in the direction $x_{i}, t$ is time, $\rho$ is fluid density, $p$ is pressure, and $v$ is kinematic viscosity. 
The Finite Volume Method (FVM) and the Pressure Implicit with Splitting of Operators (PISO) algorithm (Issa, 1986) are used to solve the equations. The FVM, as implemented in OpenFOAM, is one of the preferred methods in computational fluid dynamics (CFD) owing to the following advantages (Moukalled et al., 2016): (i) inherent conservation, (ii) allowance of unstructured polygonal meshes, and (iii) easiness of implementing boundary conditions in a non-invasive manner (since the unknown variables are evaluated at the centroids of the volume elements rather than at their boundary faces). As pointed out in Versteeg and Malalasekera (1995), the PISO algorithm is an enhanced extension of the Semi-Implicit Method for Pressure-Linked Equations (SIMPLE) algorithm. In addition, Issa et al. (1986) showed with a benchmark laminar backward-facing step problem that by using PISO over SIMPLE the computational cost reduced significantly. For the Navier-Stokes equations, the advection, diffusion and time derivative terms are discretized, respectively, using a fourth-order cubic scheme, a second-order linear scheme, and a blended scheme consisting of the second-order Crank-Nicolson scheme and a first-order Euler-implicit scheme, respectively. Since the fully centred and second-order Crank-Nicolson scheme is often unstable for complex flows, it is necessary to "off-centre" the scheme to stabilize it while retaining greater temporal accuracy than the first-order Euler-implicit scheme (Seng et al., 2017; Pedersen et al., 2017; Vukčević et al., 2016). An off-centring coefficient of 0.5 (a value of 1.0 represents the Crank-Nicolson scheme while a value of 0 represents the Euler-implicit scheme) is adopted in this study as a compromise between accuracy and stability. The same numerical approach as outlined above has been successfully adopted in Jiang et al. $(2016,2018)$ for the simulations of wake transition of a circular and a square cylinder.

\subsection{Computational mesh and boundary conditions}

The computational mesh used in Jiang et al. (2018) for the simulations of flow past a square cylinder is adopted in the present study. As sketched in Fig. 2(a), the 
computational domain size is $\left(L_{x}, L_{y}, L_{z}\right)=(60 D, 60 D, 15 D)$, with $L_{x}, L_{y}$, and $L_{z}$ being the domain sizes in the $x$-, $y$-, and $z$-directions, respectively. The boundary conditions for the computational domain are listed in Table 1 , where $\partial / \partial \mathbf{n}=0$ denotes a zero normal gradient condition. In particular, symmetry boundary conditions are applied at the top and bottom boundaries, while periodic boundary conditions are employed at the front and back boundaries that are perpendicular to the cylinder span. For the front and back boundaries, Jiang et al. (2017) demonstrated that the periodic boundary conditions are more suitable than the symmetry boundary conditions in simulating fully developed 3D flows. The periodic boundary conditions also follow the nature of the underlying instability modes which are spanwise-periodic (e.g. Henderson, 1997; Sheard et al., 2009).

(a)

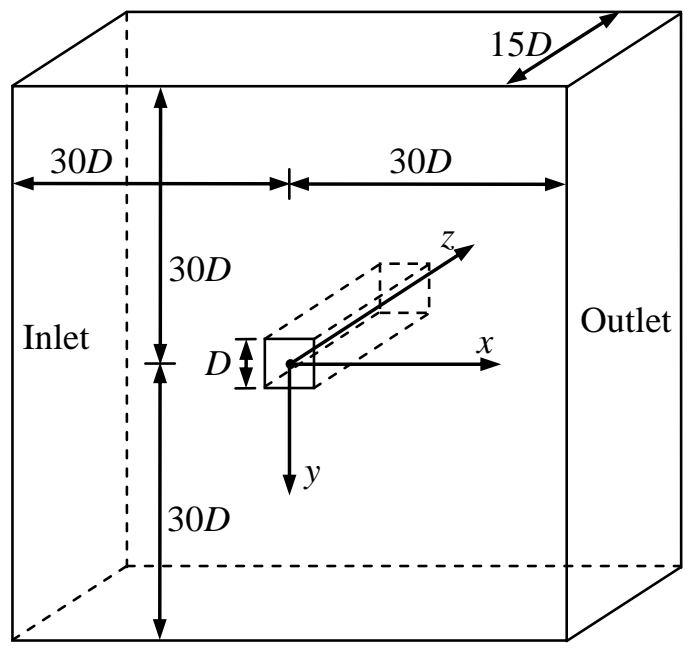




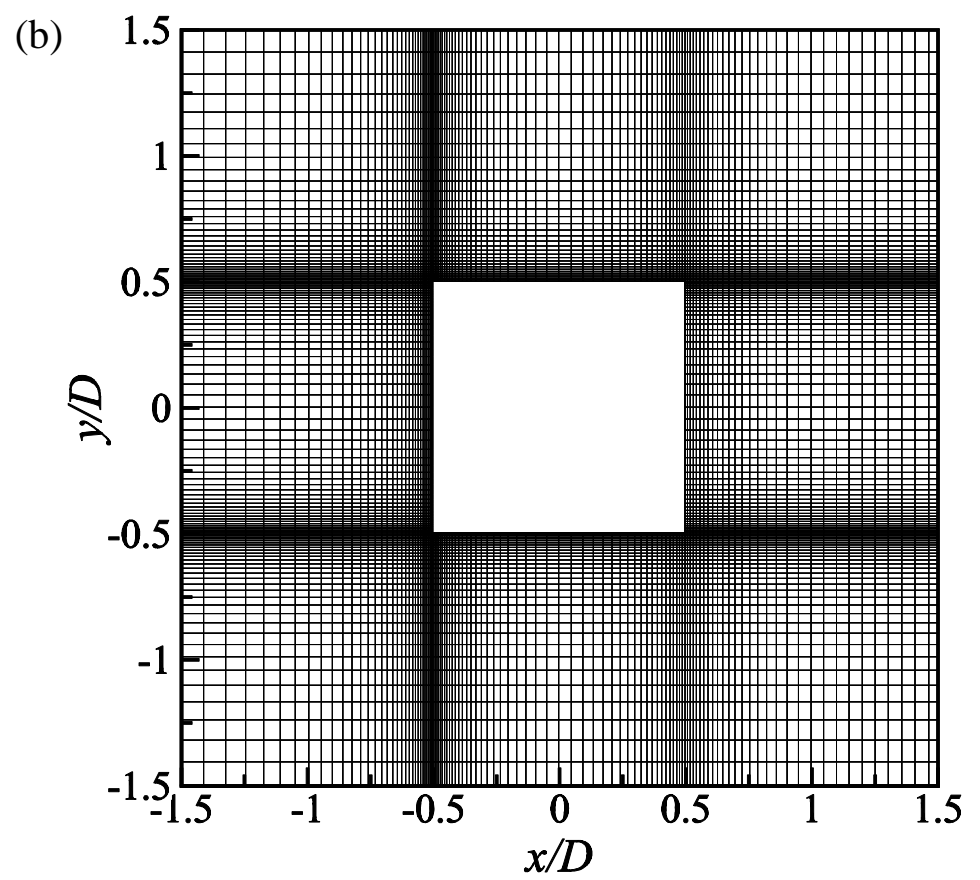

Fig. 2. (a) Schematic model of the computational domain (not to scale), and (b) Close-up view of the 2D mesh near the cylinder.

Table 1. Boundary conditions for the computational domain.

\begin{tabular}{lll}
\hline Boundary & Velocity components & Pressure \\
\hline Inlet & $u_{x}=U, u_{y}=u_{z}=0$ & $\partial p / \partial \mathbf{n}=0$ \\
Outlet & $\partial u_{i} / \partial \mathbf{n}=0$ & $p=0$ \\
Top and bottom & $\partial u_{x} / \partial \mathbf{n}=\partial u_{z} / \partial \mathbf{n}=0, u_{y}=0$ & $\partial p / \partial \mathbf{n}=0$ \\
Front and back & $u_{i}(x, y, z=0, t)=u_{i}\left(x, y, z=L_{z}, t\right)$, & $p(x, y, z=0, t)=p\left(x, y, z=L_{z}, t\right)$, \\
& $\partial u_{i} / \partial \mathbf{z}(x, y, z=0, t)=\partial u_{i} / \partial \mathrm{z}(x, y$, & $\partial p / \partial \mathbf{z}(x, y, z=0, t)=\partial p / \partial \mathrm{z}(x, y, z$ \\
& $\left.z=L_{z}, t\right)$ & $\left.=L_{z}, t\right)$ \\
Cylinder surface & $u_{i}=0$ & $\partial p / \partial \mathbf{n}=0$ \\
\hline
\end{tabular}

Some key parameters for the computational mesh are specified as follows. For the 2D mesh in the plane perpendicular to the cylinder axis (i.e. the $x-y$ plane), the number of cells around the cylinder surface is 196 . The smallest cell size at the leading edges of the cylinder is $0.005 \mathrm{D} \times 0.005 \mathrm{D}$. The cell expansion ratio in the whole domain is kept below 1.1. To capture detailed wake flow structures, a relatively high 
mesh resolution is used in the near-wake by specifying a streamwise mesh size varying linearly from $0.05 D$ at $x / D=1$ to $0.1 D$ at $x / D=7$. A close-up view of the mesh near the cylinder is shown in Fig. 2(b). The 3D mesh is constructed by replicating the $2 \mathrm{D}$ mesh along the $z$-axis with a spanwise cell size of $0.125 D$, which results in an identical mesh resolution in all planes perpendicular to the spanwise direction.

The time step size $\Delta t$ is chosen based on a Courant-Friedrichs-Lewy (CFL) limit of less than 0.5 , where the CFL number is defined as

$$
\mathrm{CFL}=\frac{|u| \Delta t}{\Delta l}
$$

where $|u|$ is the magnitude of the velocity through a cell, and $\Delta l$ is the cell size in the direction of the velocity. For $R e=100$ and 400, the time step sizes are 0.00187 and 0.00147, respectively. The temporal convergence is checked based on the $2 \mathrm{D}$ cases at $R e=50,100,200$ and 250, because the fully developed 2D flows at $R e \leq 250$ are perfectly time-periodic (Sohankar et al., 1999) and are therefore unaffected by the choice of statistical data length (i.e. the number of vortex shedding cycles used for the statistics). For the 2D cases at $R e=50,100,200$ and 250, the temporal convergence is checked by re-calculating the cases with a halved time step size (i.e. with CFL $<0.25$ ), and this results in variations of less than $0.1 \%$ for the Strouhal number and time-averaged drag coefficient, and less than $0.4 \%$ for the root-mean-square lift coefficient.

A 3D mesh convergence study has been reported separately in Jiang et al. (2018) with two variations to the above-mentioned reference mesh:

(i) A mesh refined in the $x$-y plane. This mesh has a number of cells in both $x$ - and $y$-directions 1.5 times that of the reference mesh. Specifically, the number of cells around the cylinder surface is increased by 1.5 times, while the height of the first layer of mesh next to the cylinder is reduced by 1.5 times. For this case, the time step size $\Delta t$ is also reduced by 1.5 times so as to satisfy the same CFL limit, i.e. CFL $<0.5$.

(ii) A mesh refined in the $z$-direction. The spanwise cell size is reduced from $0.125 D$ 
to $0.0625 D$.

It has been shown in Jiang et al. (2018) that the hydrodynamic forces on the cylinder for $R e=400$ (the largest $R e$ adopted in this study) calculated with the two refined meshes are very close to those calculated with the reference mesh. Specifically, the relative differences are within $0.5 \%$ for the Strouhal number and time-averaged drag coefficient, and within $3.5 \%$ for the root-mean-square lift coefficient. In addition, for the critical $R e$ values $\left(R e_{c r}\right)$ of the modes A and B wake instabilities, the relative differences in $R e_{c r}$ between those calculated with the two refined meshes and those calculated with the reference mesh are within $1 \%$. The results calculated with the reference mesh, namely $R e_{c r}=165.7$ for mode $\mathrm{A}$ and $R e_{c r}=201.1$ for mode $\mathrm{B}$, are very close to $R e_{c r}=166.6$ for mode A and $R e_{c r}=201.1$ for mode B reported in Park and Yang (2016), with relative differences of less than 1\%. Therefore, the reference mesh is used in this study.

\subsection{Data analysis methods}

In the present study, the $2 \mathrm{D}$ simulations are performed for $\operatorname{Re}$ up to 265 , since above which the vortex shedding process becomes irregular due to the restriction of a 2D computational domain (Sohankar et al., 1999). For $R e \geq 270$, it is found that the vortex shedding process no longer follows the Kármán vortex street pattern, and the time-averaged lift force is not zero. However, these features are not observed for the simulations with a 3D computational domain. The 3D simulations are therefore performed for $\operatorname{Re}$ up to 400 .

The hydrodynamic forces on the cylinder, including the drag coefficient $\left(C_{D}\right)$, the lift coefficient $\left(C_{L}\right)$, and the Strouhal number $(S t)$, are defined as:

$$
\begin{aligned}
& C_{D}=\frac{F_{D}}{\frac{1}{2} \rho U^{2} D L_{z}} \\
& C_{L}=\frac{F_{L}}{\frac{1}{2} \rho U^{2} D L_{z}} \\
& S t=\frac{f_{L} D}{U}
\end{aligned}
$$


where $F_{D}$ and $F_{L}$ are the drag force and lift force integrated over the entire cylinder surface, respectively, and $f_{L}$ is the frequency of the fluctuating lift force. The time-averaged drag and lift coefficients are denoted as $\overline{C_{D}}$ and $\overline{C_{L}}$, respectively. The root-mean-square lift coefficient $C_{L}{ }^{\prime}$ is defined as:

$$
C_{L}^{\prime}=\sqrt{\frac{1}{N} \sum_{i=1}^{N}\left(C_{L, i}-\overline{C_{L}}\right)^{2}}
$$

where $N$ is the number of values in the time-history.

In addition, the time-averaged pressure coefficient $\left(C_{p}\right)$ at a specific point on the cylinder surface is defined as:

$$
C_{p}=\frac{p_{c y l}-p_{\infty}}{\frac{1}{2} \rho U^{2}}
$$

where $p_{c y l}$ is the time-averaged pressure at this point, and $p_{\infty}$ is the reference pressure at the inlet of the domain. The base pressure coefficient $\left(C_{p b}\right)$ is defined as:

$$
C_{p b}=\frac{p_{b}-p_{\infty}}{\frac{1}{2} \rho U^{2}}
$$

where $p_{b}$ is the time-averaged pressure at the rear stagnation point of the cylinder, namely $(x / D, y / D)=(0.5,0)$.

Prior to the onset of vortex shedding of $R e=46$, the $\overline{C_{D}}$ and $C_{p}$ values are obtained from the fully developed steady flow, while the fluctuations of the hydrodynamic forces are equal to zero. For $R e=46-265$, since the fully developed 2D flows are time-periodic, the hydrodynamic forces are calculated based on the time-histories of a complete vortex shedding cycle. Beyond the onset of the secondary wake instability of $R e=165.7$, the fully developed 3D flows become irregular. For each 3D case, the simulation is run for at least 1000 non-dimensional time units (defined as $t^{*}=t U / D$ ) to ensure that the flow has become fully developed. After that, at least 800 non-dimensional time units ( 120 vortex shedding cycles) of the fully developed flow are used to calculate the statistical stationary hydrodynamic forces on the cylinder. For $3 \mathrm{D}$ flows, $f_{L}$ is determined as the peak frequency derived from the fast Fourier transform (FFT) of the time-history of $C_{L}$. 
In addition to the hydrodynamic forces on the cylinder, the flow field around the cylinder will also be examined. The time-averaged streamwise, transverse and spanwise velocities are denoted as $\overline{u_{x}}, \overline{u_{y}}$ and $\overline{u_{z}}$, respectively, while the root-mean-square streamwise, transverse and spanwise velocities are calculated as:

$$
\begin{aligned}
& u_{x}^{\prime}=\sqrt{\frac{1}{N} \sum_{i=1}^{N}\left(u_{x, i}-\overline{u_{x}}\right)^{2}} \\
& u_{y}^{\prime}=\sqrt{\frac{1}{N} \sum_{i=1}^{N}\left(u_{y, i}-\overline{u_{y}}\right)^{2}} \\
& u_{z}^{\prime}=\sqrt{\frac{1}{N} \sum_{i=1}^{N}\left(u_{z, i}-\overline{u_{z}}\right)^{2}}
\end{aligned}
$$

For a 3D flow, at least 800 non-dimensional time units of the fully developed flow are used to calculate the time-averaged and root-mean-square 3D flow fields.

Ideally, for a 3D flow the hydrodynamic forces at different spanwise locations would be identical when the statistical period is sufficiently long. When a finite statistical period is used (even for more than 120 vortex shedding cycles), the $3 \mathrm{D}$ hydrodynamic forces at different spanwise locations may be slightly different, and the span-averaged values can be used to improve the accuracy. Hence equations (2.4) (2.7) give the span-averaged hydrodynamic forces. By using equations (2.4) - (2.7), the hydrodynamic forces for the 3D flow at $R e=400$ calculated with the statistical data range of at least 800 non-dimensional time units are very close to those calculated with the second half of the data range (of at least 400 non-dimensional time units), with relative differences of less than $0.3 \%$ for $S t$ and $\overline{C_{D}}$, and less than $2 \%$ for $C_{L}^{\prime}$ (Jiang et al., 2018).

Similarly, for a 3D flow the wake flow characteristics (such as the formation length and the wake width) at different spanwise locations may be slightly different when using a finite statistical period. To approximate to the ideal results, the time-averaged/root-mean-square 3D flow field is further averaged over the spanwise direction to form a time-averaged/root-mean-square 2D flow field (called span-averaged 3D flow hereafter). Such a 2D flow field can be used to determine the 
span-averaged flow characteristics of the corresponding 3D flow.

Comparisons of the flow characteristics of flow past a square cylinder and a circular cylinder are also included in some of the analyses. For the DNS results of flow past a circular cylinder reported in this study, the computational meshes used in Jiang et al. (2016) and Jiang and Cheng (2017) are adopted. Convergence checks of the meshes for a circular cylinder have been reported separately in Jiang et al. (2016) with $R e=220$ and 300 and in Jiang and Cheng (2017) with $R e=400$ and 1000 .

\section{Numerical results}

\subsection{Variations of flow properties with $R e$}

Fig. 3 shows the variations of some flow properties with $R e$, including the time-averaged drag coefficient, the root-mean-square lift coefficient, the base pressure coefficient, the maximum 2D Reynolds stress $u_{x}{ }^{\prime} u_{y}{ }^{\prime}$ in the flow field, the formation length, and the Strouhal number. The formation length is calculated as the streamwise distance between the rear surface of the cylinder and the wake stagnation point, as sketched in Fig. 4.

(a)

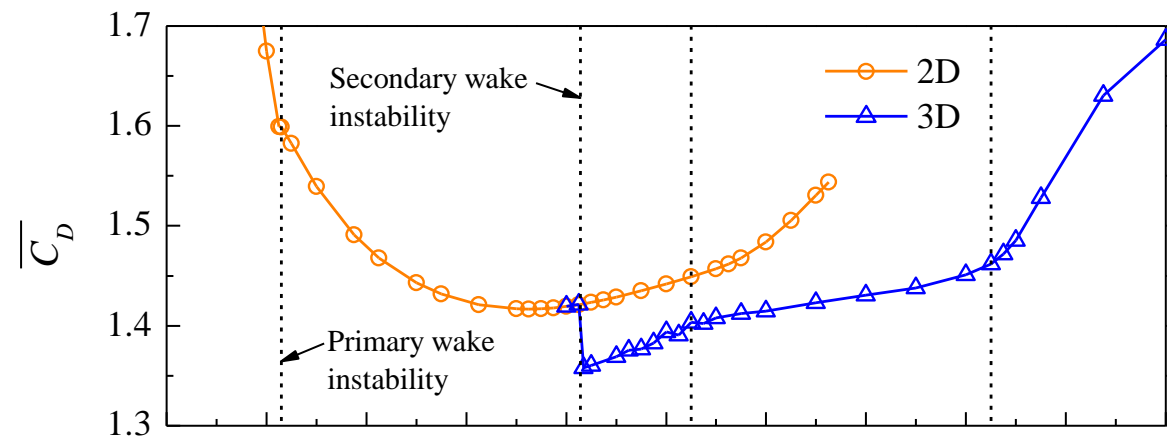

(b)

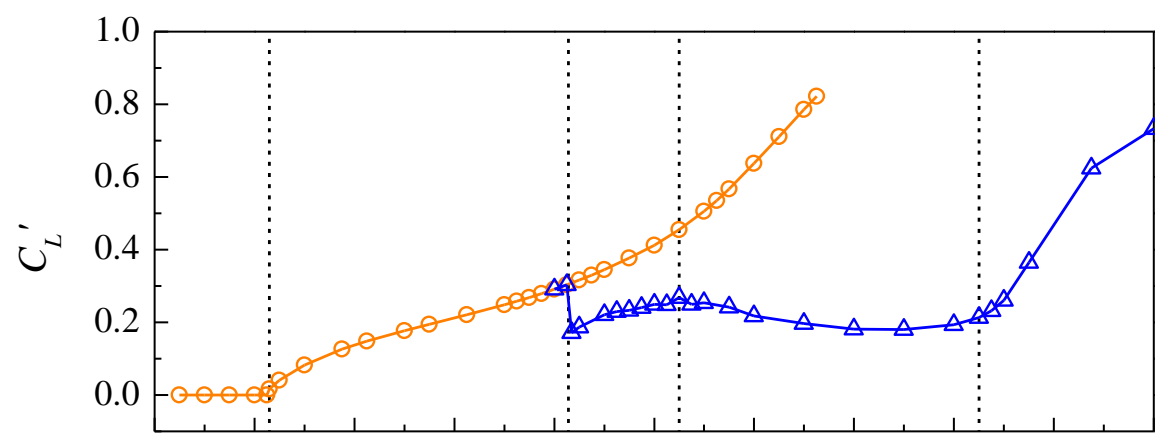


(c)

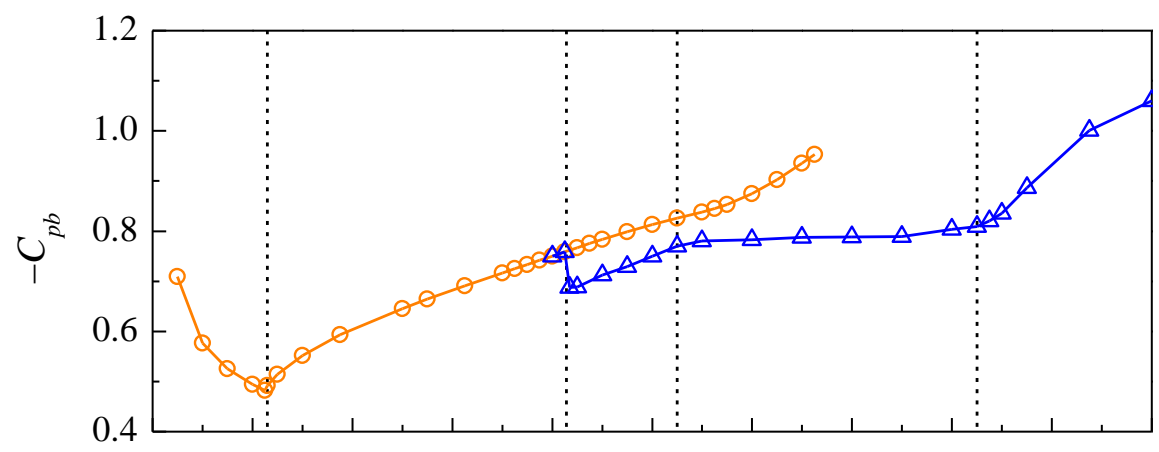

(d)

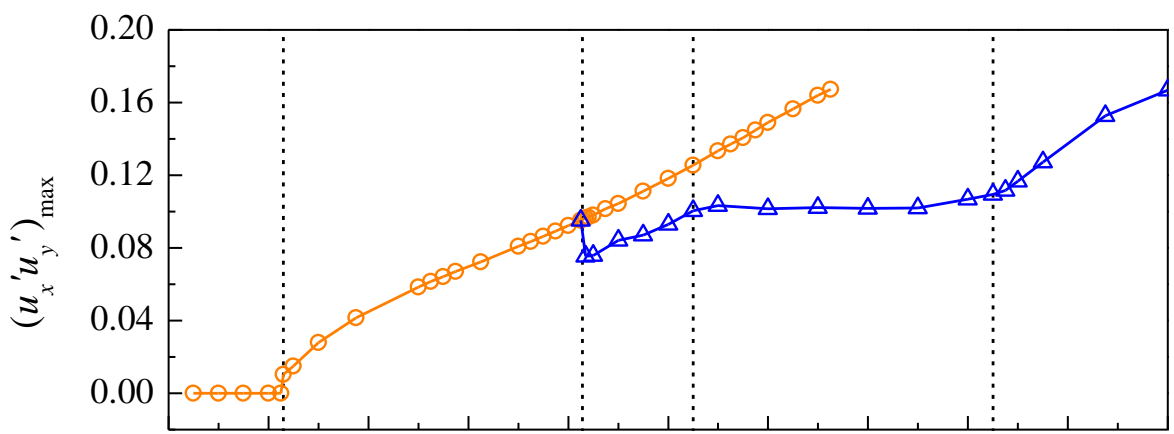

(e)

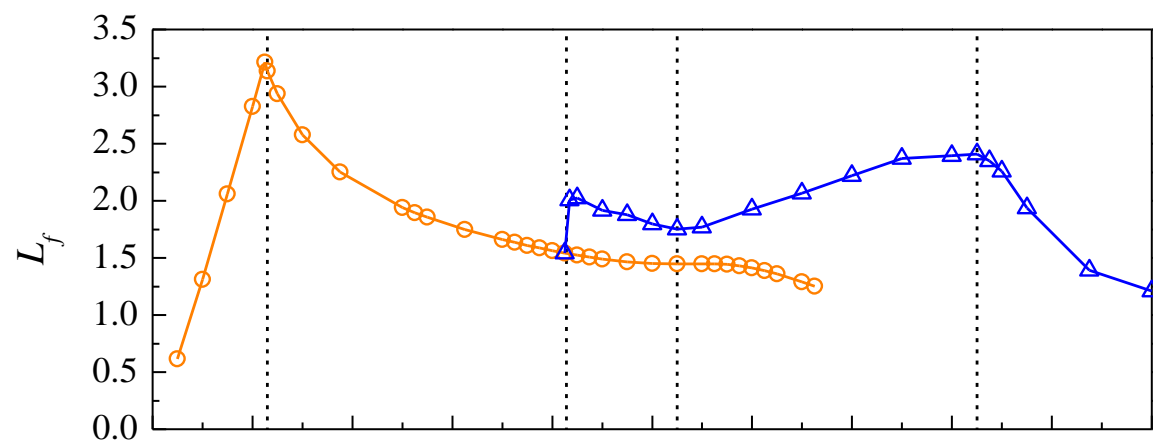

(f)

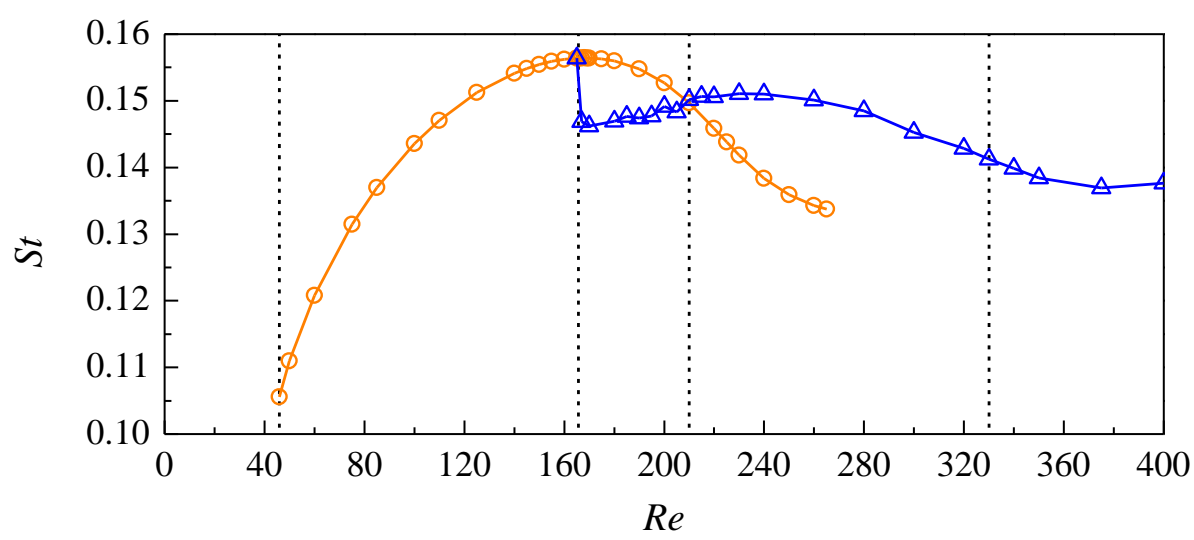

Fig. 3. Variations of flow properties with $R e$ : (a) the time-averaged drag coefficient, (b) the root-mean-square lift coefficient, (c) the base pressure coefficient, (d) the maximum $u_{x}{ }^{\prime} u_{y}{ }^{\prime}$ in the flow field, (e) the formation length, and (f) the Strouhal number. The vertical dashed lines mark the critical points at $R e=46,165.7,210$ and 330 . 


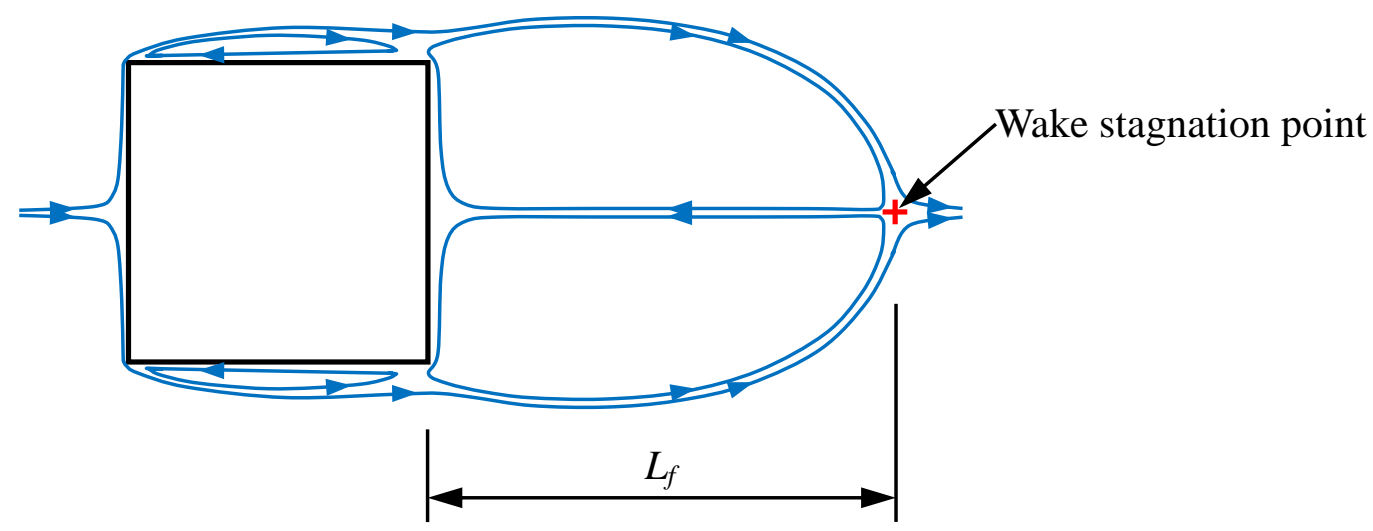

Fig. 4. A sketch of the definition of the formation length $L_{f}$. The blue lines represent the streamlines of the time-averaged flow, where two recirculation regions are observed in the near wake of the cylinder.

The variations of flow properties with $R e$ shown in Fig. 3 reveal four critical points for $R e$ up to 400, as marked by the vertical dashed lines in Fig. 3. The first two critical points at $R e=46$ and 165.7 are induced by the primary and secondary wake instabilities, respectively. It is not surprising that significant changes in the flow pattern, such as the onset of vortex shedding and the onset of three-dimensionality, would result in sudden variations in the values of the flow properties. Fig. 5 shows some instantaneous vorticity fields for $R e$ in different wake transition regimes. The onset of three-dimensionality is evidenced by the emergence of streamwise vortices for $R e>165.7$. Beyond the secondary wake instability, two more critical points are observed at $R e$ of approximately 210 and 330. These two critical points are hardly identified in previous studies (see Fig. 1b). 
(a)

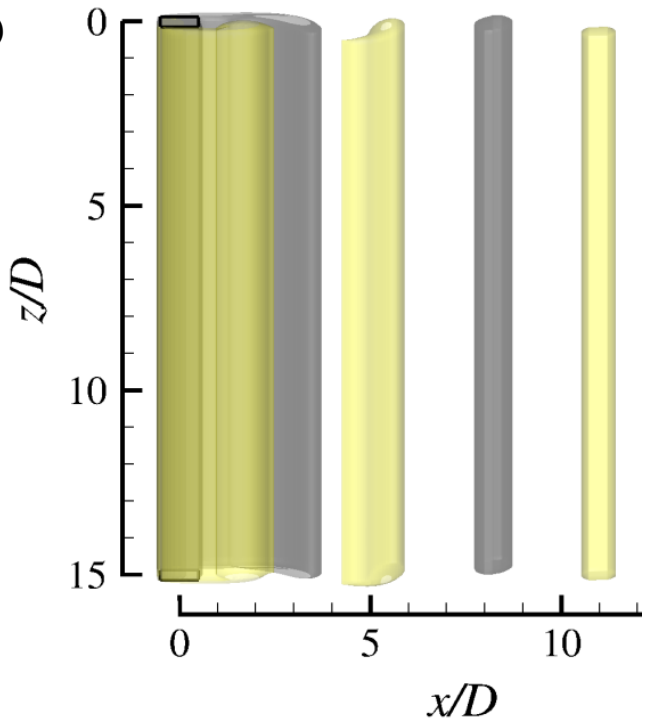

(c)

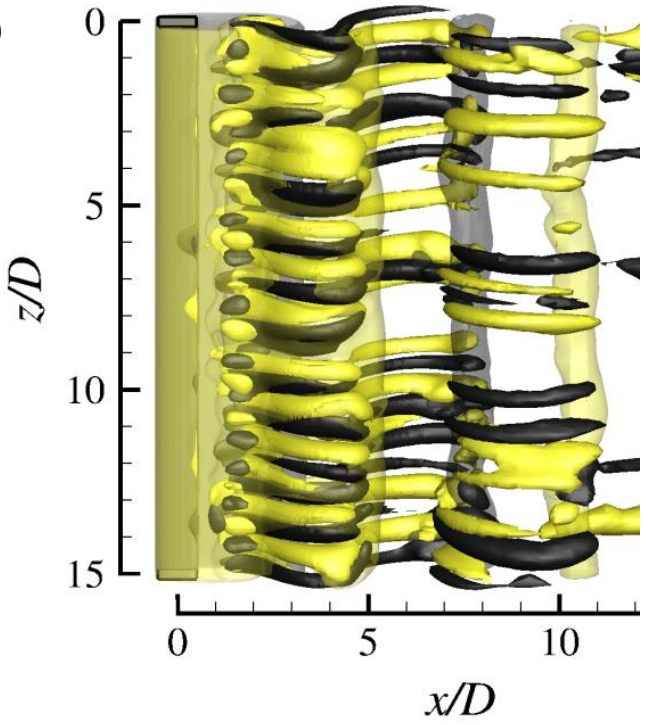

(b)

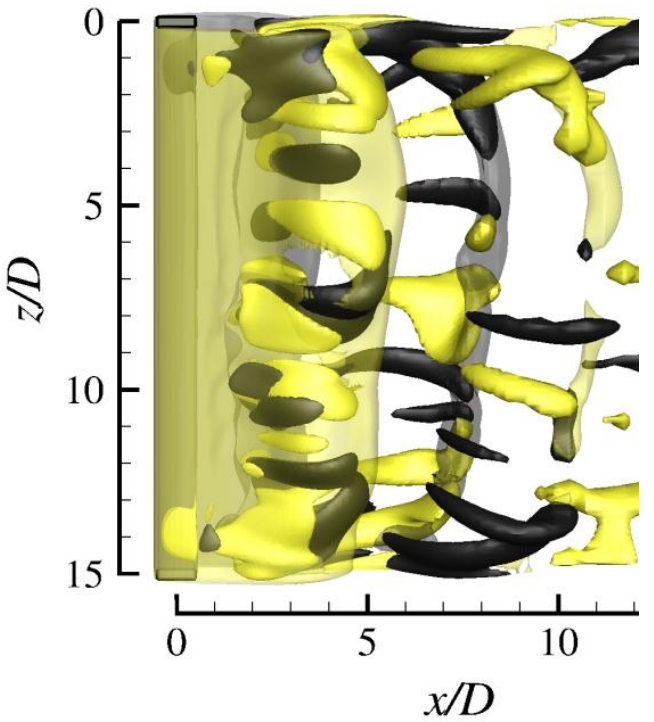

(d)

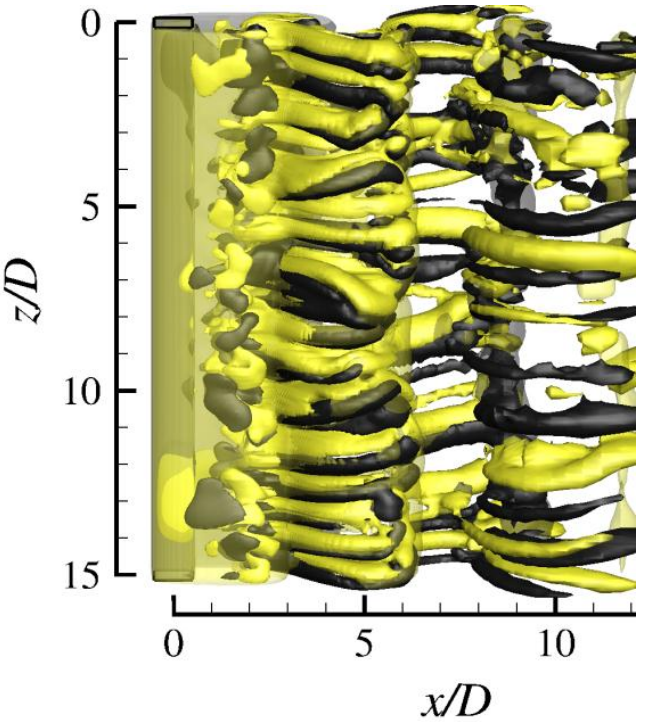

Fig. 5. Instantaneous vorticity fields in the near wake of a square cylinder for (a) $R e=$ 165 (with only spanwise vortices), (b) $R e=170$ (with mode A streamwise vortices and vortex dislocations), (c) $R e=220$ (with relatively ordered mode B streamwise vortices), and (d) $R e=330$ (with disordered mode B streamwise vortices). The translucent iso-surfaces represent spanwise vortices with $\omega_{z}= \pm 1.0$, while the opaque iso-surfaces represent streamwise vortices with $\omega_{x}= \pm 0.5$. Dark grey and light yellow denote positive and negative vorticity values, respectively. The flow is from left to right past the cylinder on the left.

Some variation features observed in Fig. 3 are summarised below, and some of which will be further investigated in sections $3.2-3.4$. 
(i) Similar variation trends are observed for the base pressure coefficient shown in Fig. 3(c) and the maximum 2D Reynolds stress shown in Fig. 3(d). This feature is a result of the balance of forces from pressure and shear stress in the recirculation region, as is also observed for the case of a circular cylinder (Williamson, 1996a). In addition, the variation trends for the formation length shown in Fig. 3(e) are opposite to those of the base pressure coefficient and maximum 2D Reynolds stress, which suggests that an increase in the forces in the recirculation region would lead to a reduction in the size of the recirculation region, and vice versa.

(ii) At the onset of the primary wake instability of $R e=46$, a discontinuity in the gradient of variation with $R e$ is observed for each of the flow properties shown in Fig. 3. For $R e \geq 46$, a monotonic increase or decrease is observed for each of the 2D flow properties shown in Fig. 3(b-e). However, for the time-averaged drag coefficient shown in Fig. 3(a) a local minimum is observed at $R e=144$, while for the Strouhal number shown in Fig. 3(f) a local maximum is observed at $R e=168$. The physical mechanisms for these two extreme values are investigated in sections 3.2 and 3.3 , respectively.

(iii) The two critical points beyond the secondary wake instability (at $R e \sim 210$ and 330) are observed for all the flow properties shown in Fig. 3 except for the Strouhal number shown in Fig. 3(f). The different behaviour for the 3D St-Re relationship is investigated in section 3.4.

\subsection{The drag coefficient}

To further examine the variation of the time-averaged drag coefficient with $R e$ shown in Fig. 3(a), the total drag is separated into the pressure and viscous components in Fig. 6(a). To facilitate comparison, the pressure and viscous drag coefficients for a circular cylinder are shown in Fig. 6(b). It is seen in Fig. 6(b) that the coefficients calculated with the present 2D DNS are in good agreement with the results reported in Henderson (1995). As stated in Henderson (1995), as $R e$ exceeds the onset of the primary wake instability, the pressure drag coefficient starts to 
increase while the viscous drag coefficient continues to decrease (Fig. 6b). The local minimum in the total drag coefficient at $R e \sim 150$ is due to combined effects of the pressure and viscous components. Similar variation trends are also observed in Fig. 6(a) for flow past a square cylinder, which explains the local minimum in the total drag at $R e=144$.

For the case of flow past a square cylinder, an interesting feature observed in Fig. 6(a) is that the viscous drag coefficient decreases to negative values for $R e \geq 154$, and consequently the total drag coefficient is smaller than the pressure drag coefficient. The reason for the negative viscous drag is that the sizes of the recirculation regions on the upper and lower sides of the square cylinder expand with increasing $R e$, such that the upper and lower surfaces of the cylinder are increasingly covered with backward flow rather than forward flow. For $R e>150$ the backward flow covers almost entirely the upper and lower surfaces of the cylinder. In contrast, for flow past a circular cylinder the flow separates at the downstream half of the cylinder surface (Wu et al., 2004), and the forward flow prior to the separation point is stronger than the backward flow after separation, such that the viscous drag integrated over the cylinder surface is positive (Fig. 6b). 
(a)

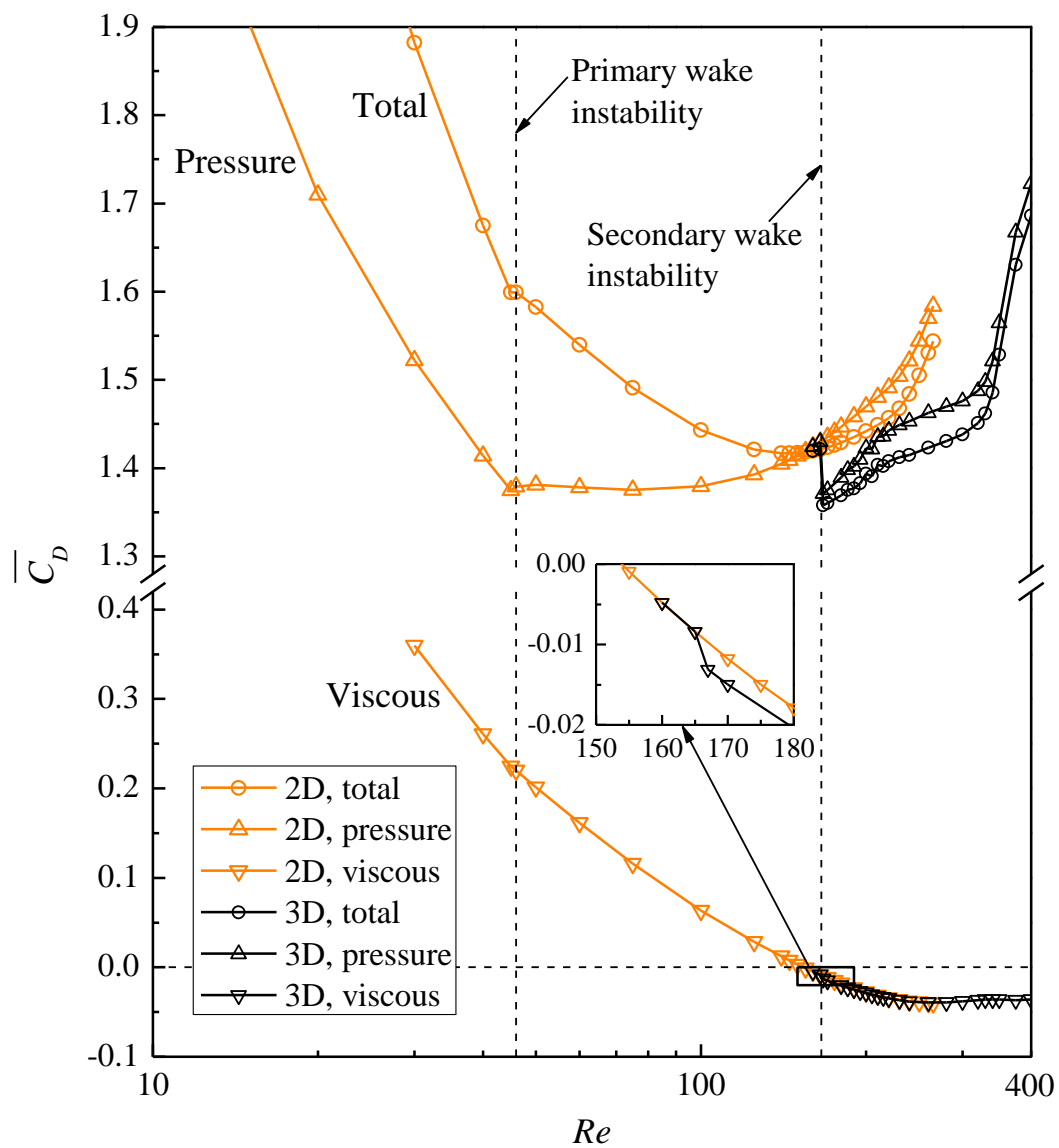

(b)

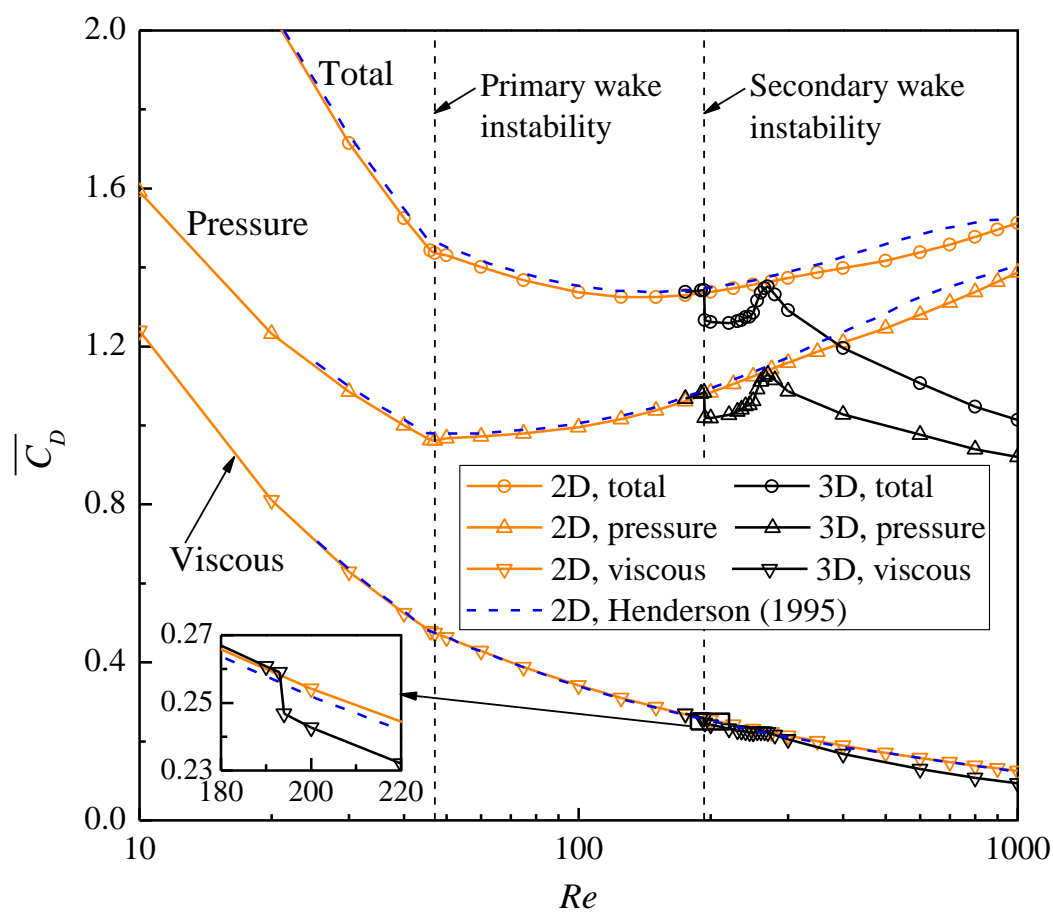

Fig. 6. Variations of the pressure and viscous drag coefficients with $R e$ for (a) flow past a square cylinder, and (b) flow past a circular cylinder.

It is also seen in Fig. 6 that the pressure drag coefficient for a square cylinder is 
larger than that for a circular cylinder. This is mainly because the front surface of the square cylinder is perpendicular to the incoming flow, such that the streamwise velocity at the front of the cylinder (except for the areas very close to the edges) reduces to almost zero. A comparison of the time-averaged pressure coefficient on a square cylinder and a circular cylinder for $R e=100$ is shown in Fig. 7(a,b). To reveal better the pressure drag in the streamwise direction, the streamwise component of the pressure coefficient is shown in Fig. 7(c,d). As shown in Fig. 7(c), the majority of the front surface of the square cylinder (except for the areas very close to the edges) is covered with a positive pressure, which is due to a sudden changeover of the velocity from the streamwise direction to the transverse direction. In contrast, for a circular cylinder the positive pressure is observed only within approximately $\pm 39^{\circ}$ from the front point of the cylinder (Fig. 7d), within which a sudden changeover of the direction of the velocity takes place.

(a)

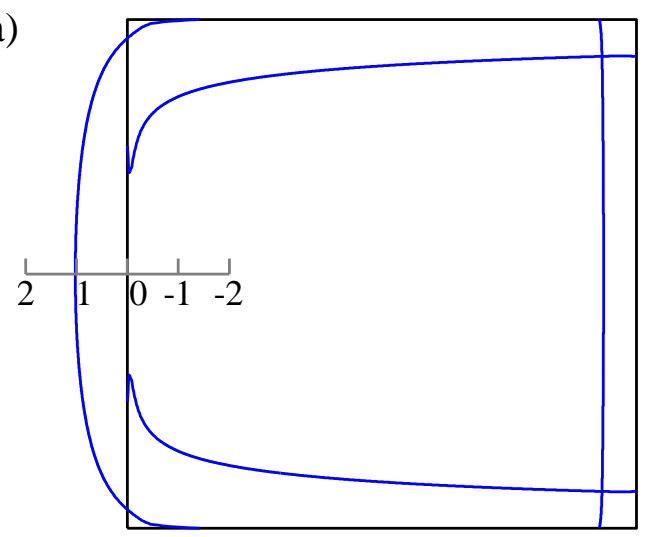

(c)

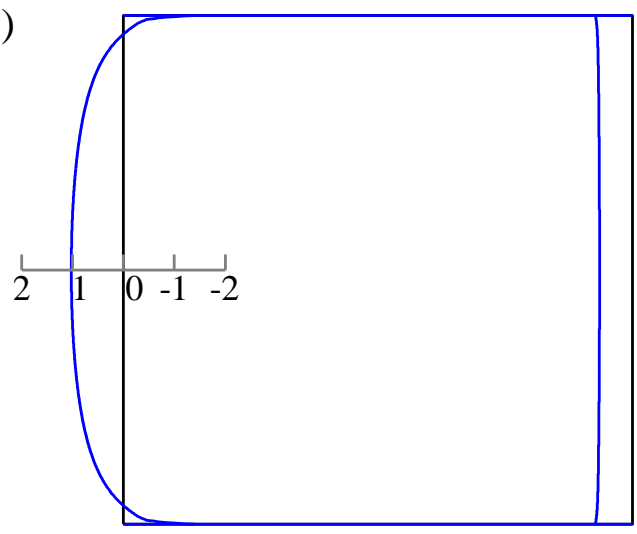

(b)

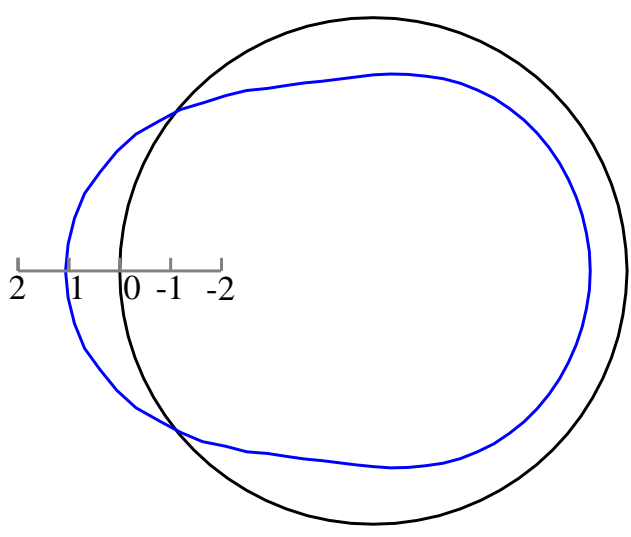

(d)

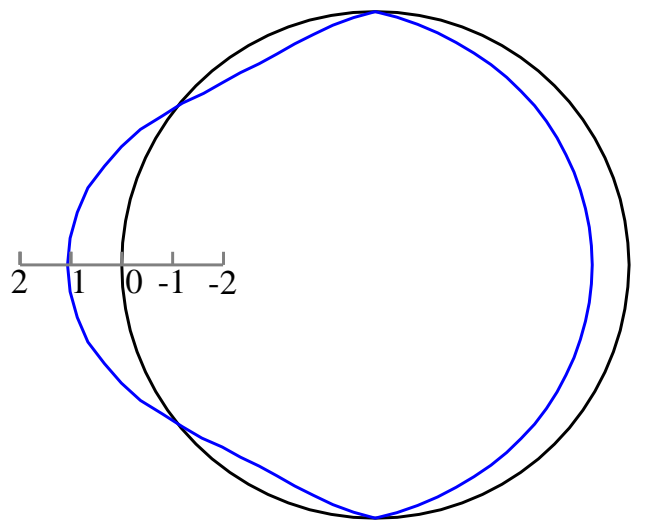

Fig. 7. Time-averaged pressure coefficient on the cylinder for $R e=100$ for (a) flow 
past a square cylinder, and (b) flow past a circular cylinder. In addition, (c) and (d) show the streamwise component of the pressure coefficient for a square and a circular cylinder, respectively. The cylinder surface is shown in black while the pressure coefficient is plotted in blue. The flow is from left to right.

\subsection{The 2D $S t-R e$ relationship}

For the 2D St-Re relationship, a major difference between a circular cylinder and a square cylinder is that for a circular cylinder $S t$ increases monotonically with increase in $R e$ up to at least $R e=1000$ (see, e.g., Henderson, 1997), whereas for a square cylinder the $S t-R e$ curve peaks at $R e=168$ (Fig. 3f).

The physical reason is analysed below. Roshko (1955) suggested that the Strouhal number of a bluff body would scale better on the velocity at separation $\left(U_{s}\right)$ and the wake width $\left(D^{\prime}\right)$, rather than simply on $U$ and $D$. The Strouhal number based on such a definition was called the wake Strouhal number $\left(S t^{*}\right)$ in Roshko (1955), i.e.

$$
S t^{*}=f_{L} \frac{D^{\prime}}{U_{s}}=S t \frac{U}{U_{s}} \frac{D^{\prime}}{D}
$$

In the present study, the $U_{s}$ and $D^{\prime}$ values for a square cylinder are determined based on the spanwise vorticity contours of the time-averaged flow field. The spanwise vorticity $\omega_{z}$ is defined in a non-dimensional form:

$$
\omega_{z}=\left(\frac{\partial u_{y}}{\partial x}-\frac{\partial u_{x}}{\partial y}\right) \frac{D}{U}
$$

As illustrated in Fig. 8, $D^{\prime}$ is chosen as the largest vertical distance between the positive and negative $\omega_{z}$ peaks. This is because such a distance reveals directly the cross-flow extent for the vortices to roll up to trigger vortex shedding (Jiang and Cheng, 2017). On the other hand, $U_{s}$, which serves as a representative of the regional flow rate prior to the rolling up of the vortices, is chosen as the mean streamwise velocity along the velocity profile shown by the solid vertical line located at $x / D=$ 0.465 (Fig. 8). The streamwise location is chosen at $x / D=0.465$ because it is very close to the saddle point between the recirculation regions on the upper/lower side of 
the cylinder and in the wake region, which will be further explained later on in this section. The length of each vertical line is twice the distance between the peak point of $\omega_{z}$ at $x / D=0.465$ (marked by a solid dot on the vertical line in Fig. 8) and the cylinder surface. The $U_{s}-R e$ and $D^{\prime}-R e$ relationships based on the present method are shown in Fig. 9(a) and Fig. 9(b), respectively. The corresponding $S t^{*}-R e$ relationship is shown in Fig. 10(a).

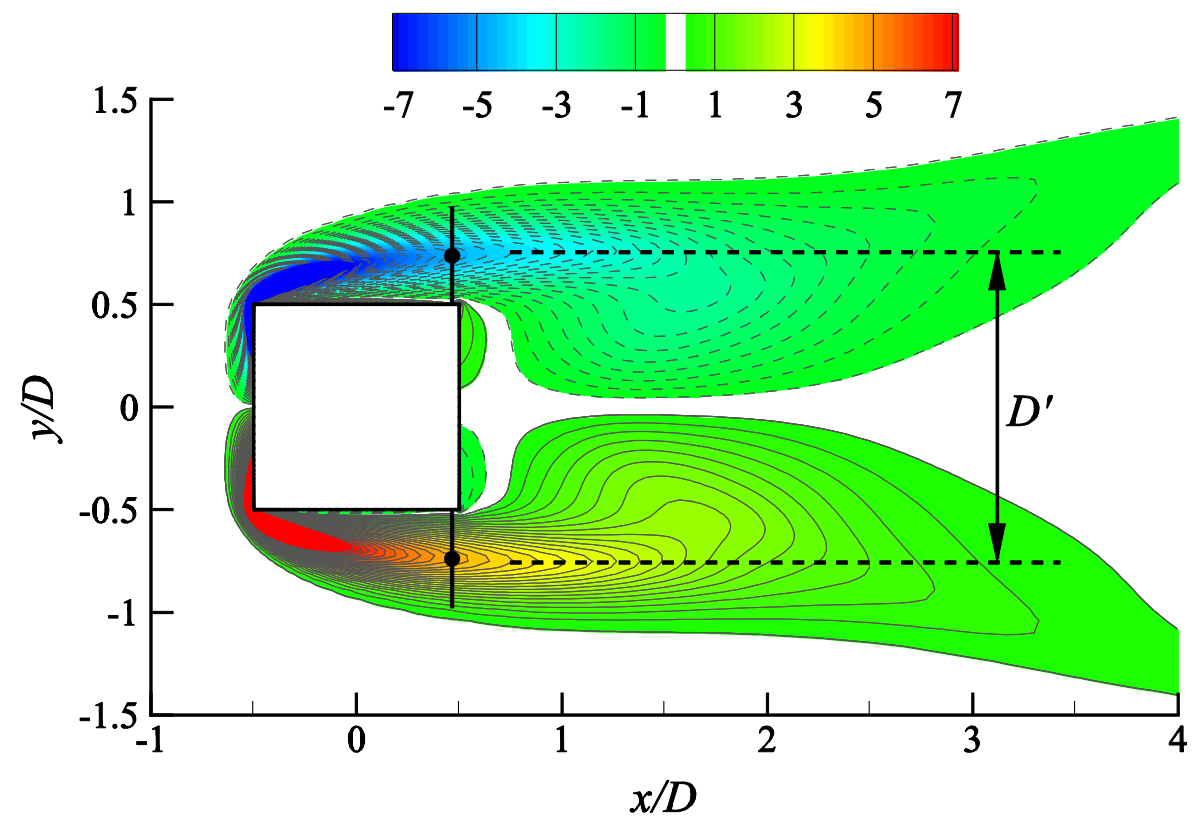

Fig. 8. Determinations of $U_{s}$ and $D^{\prime}$ based on the spanwise vorticity contours of the time-averaged 2D flow field at $R e=168$. Positive and negative contours are marked by solid and dashed lines, respectively. 
(a)

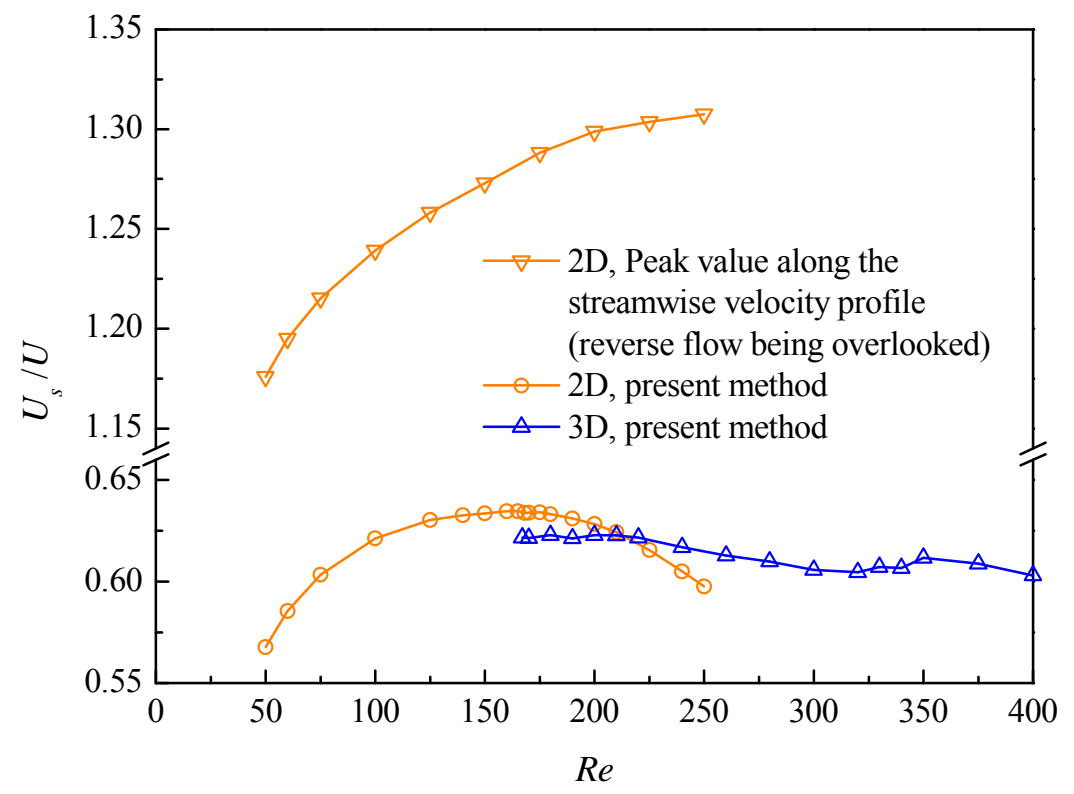

(b)

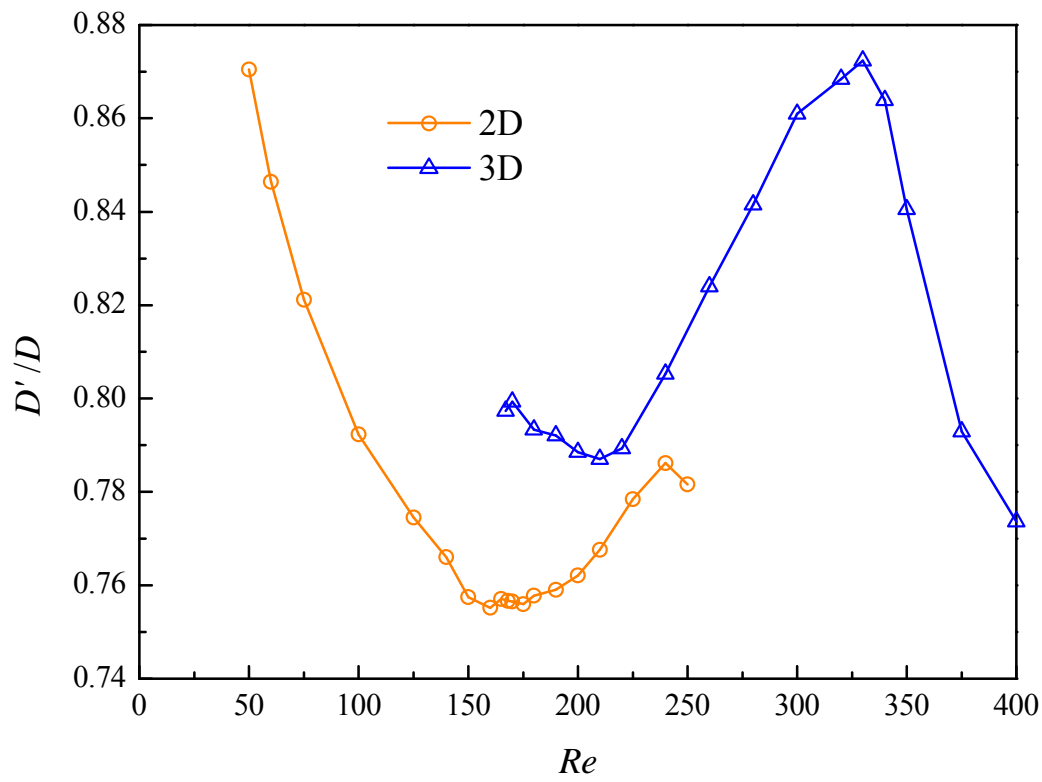

Fig. 9. (a) The $U_{s}-R e$ relationship, and (b) the $D^{\prime}-R e$ relationship, determined based on the spanwise vorticity contours of the time-averaged flow field. 
(a)

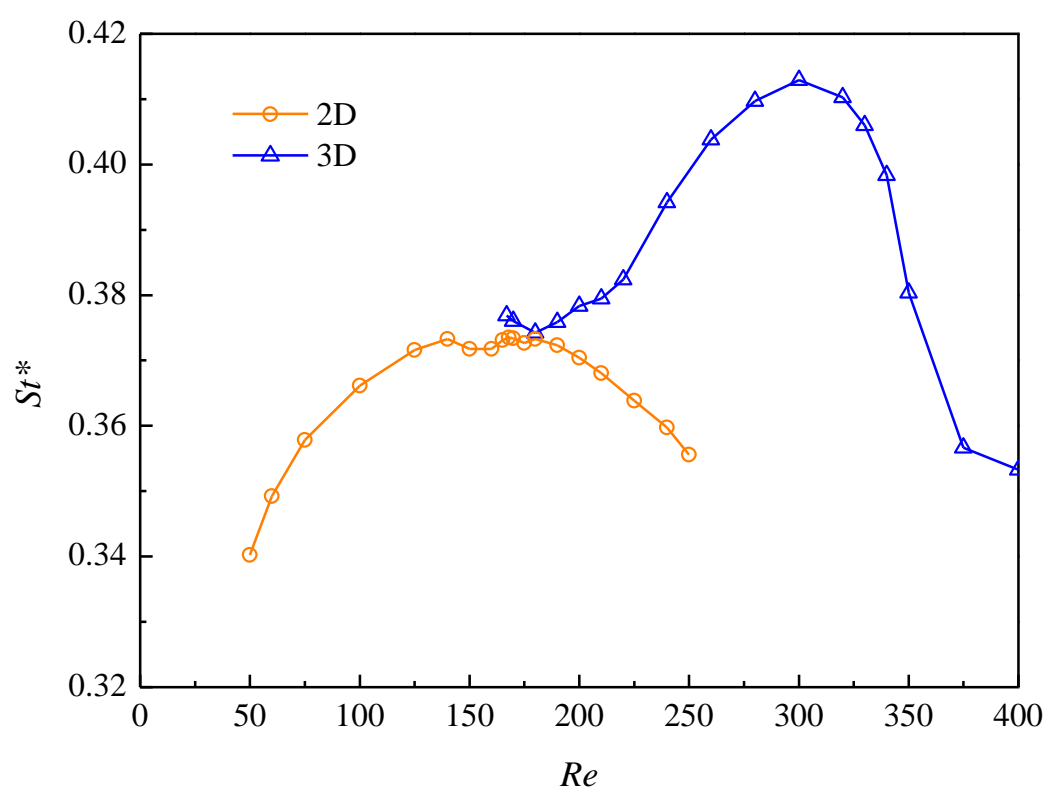

(b)

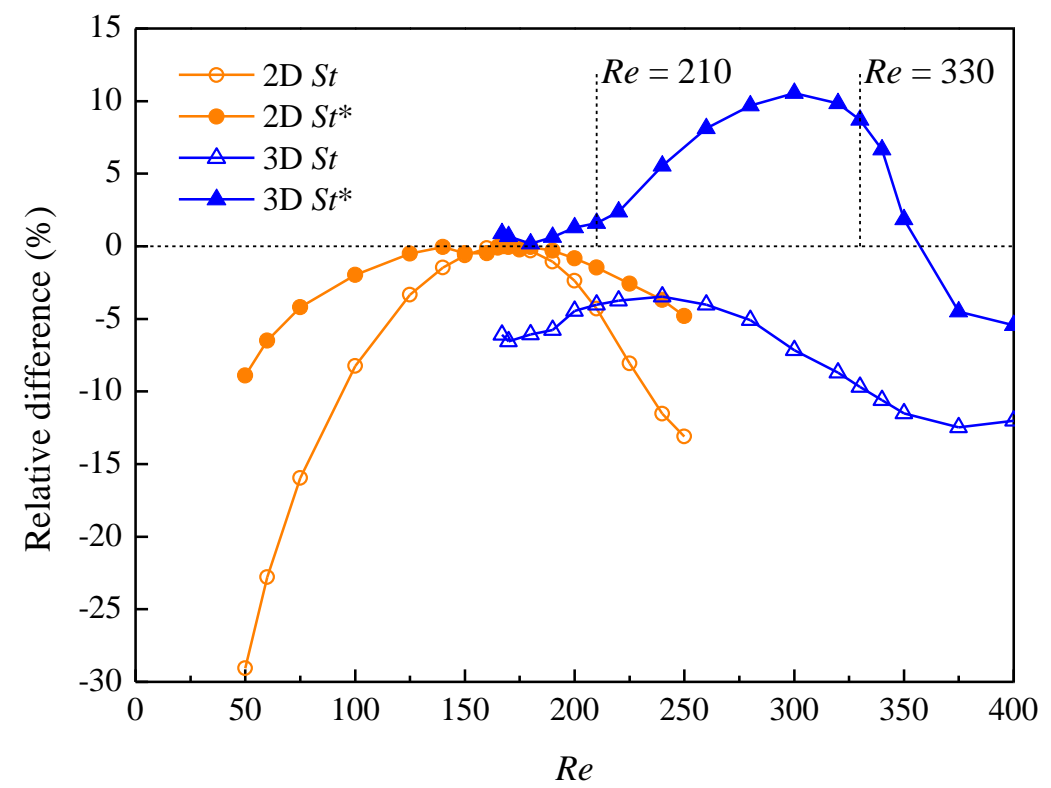

Fig. 10. (a) The 2D and 3D $S t^{*}-R e$ relationships over the laminar and 3D wake transition regimes. (b) Comparison of the uniformness of the $S t$ and $S t^{*}$ values, shown by the relative differences of the $S t$ values with respect to the $2 \mathrm{D} S t$ value at $R e=168$, and the relative differences of the $S t^{*}$ values with respect to the $2 \mathrm{D} S t^{*}$ value at $R e=$ 168.

To examine the uniformity of the $S t$ and $S t^{*}$ values over the range of Re, Fig. 10(b) shows (i) the relative differences of the $S t$ values with respect to the 2D $S t$ value at $R e$ $=168$, and (ii) the relative differences of the $S t^{*}$ values with respect to the $2 \mathrm{D} S t^{*}$ value at $R e=168$. It is seen in Fig. 10(b) that the $2 \mathrm{D} S t^{*}$ values (with relative 
differences smaller than 10\%) are indeed more uniform than the 2D St values (with relative differences up to $29 \%$ ). This suggests that the vortex shedding frequency scales better on $U_{s}$ and $D^{\prime}$ rather than on $U$ and $D$. The inconsistency of the 2D $S t^{*}$ values may be due to some secondary factors such as the selection criterion for $U_{s}$ in representing the regional flow prior to vortex shedding, and the redistribution of the velocity profile before the rolling up of the vortices.

As shown in Fig. 9, the peak of the $S t-R e$ curve at $R e=168$ (Fig. 3f) is due to the peak of the $U_{s}-R e$ curve and the corresponding trough of the $D^{\prime}-\operatorname{Re}$ curve (a larger $U_{s}$ leads to a thinner shear layer and thus a smaller $D^{\prime}$ ) at $R e$ very close to 168 . Based on flow visualization, it is found that the decrease in $U_{s}$ for $R e>168$ (Fig. 9a) is due to the emergence of a reverse flow from the wake recirculation region to the upper and lower sides of the cylinder past the two trailing edges of the cylinder, as illustrated with the streamlines of the time-averaged 2D flow field in Fig. 11. The reverse flow is not observed at $R e=166$ (Fig. 11a) but emerges for $R e \geq 167$ (e.g. Fig. 11b). It is also noticed that the streamlines shown in Fig. 11 may slightly dive through the corner of the square cross-section, since the streamlines are drawn based on the time-averaged flow field rather than an instantaneous flow field. Nevertheless, the time-averaged velocity near the ends of the streamlines is well below $0.01 U$. 
(a)

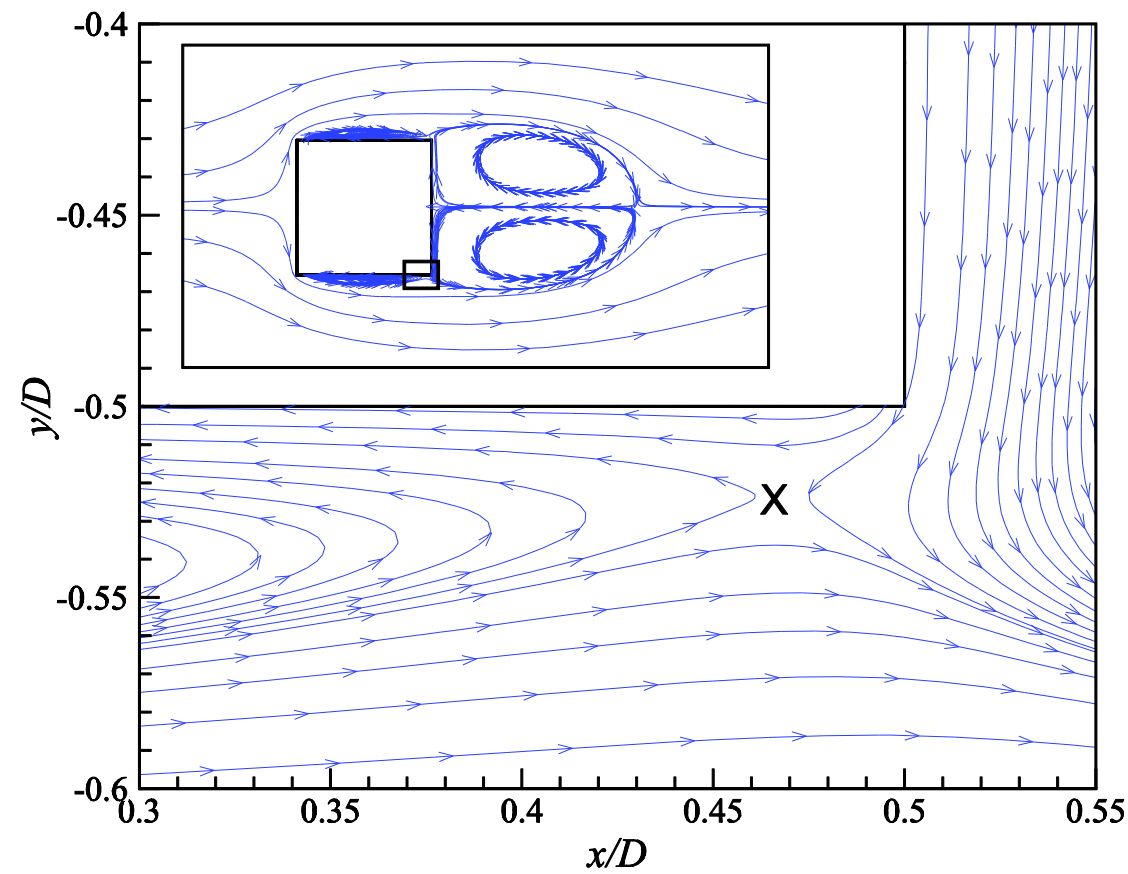

(b)

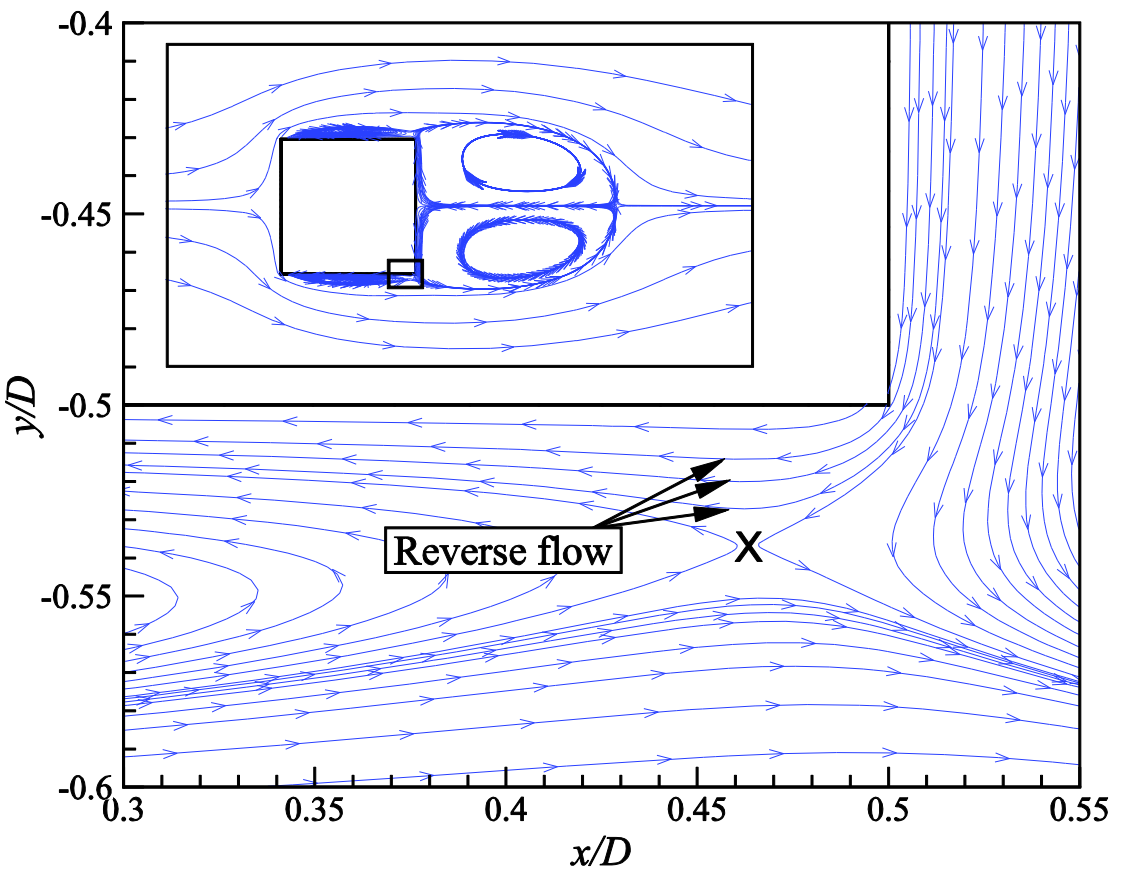

Fig. 11. Streamlines of the time-averaged 2D flow field for (a) $R e=166$, and (b) $R e=$ 175. The streamlines are shown at near the lower right corner of the square cylinder, as marked by a rectangular frame in the inset. The saddle point is marked by a cross.

It is noted that the streamwise location for the calculation of $U_{s}$ is chosen at $x / D=$ 0.465 (which is very close to the saddle point shown in Fig. 11) so as to properly consider the reverse flow in the calculation of $U_{s}$. It is also worth noting that the $U_{s}$ 
value for a square cylinder is chosen as the mean streamwise velocity along a certain vertical range at $x / D=0.465$, rather than the velocity at a particular point. This is because the velocity profile still redistributes as the flow travels from the separation point to the point where vortices start to roll up. The use of the mean streamwise velocity for $U_{s}$ allows the reverse flow to be considered. For the case of a circular cylinder, however, the peak value along the streamwise velocity profile has been used to represent $U_{s}$ (Williamson and Brown, 1998; Jiang and Cheng, 2017), since there is no reverse flow prior to the wake recirculation region. For the case of a square cylinder, if the peak value along the streamwise velocity profile is used to represent $U_{s}$, the reverse flow would be overlooked, since such a $U_{s}$ value increases monotonically with increasing $R e$ (also shown in Fig. 9(a)).

\subsection{The 3D $S t-R e$ relationship}

The $S t^{*}$ values for the (span-averaged) 3D flows are also examined. The $U_{s}-R e$, $D^{\prime}-R e$ and $S t^{*}-R e$ relationships for the 3D flows are plotted in Fig. 9 and Fig. 10(a). Fig. 10(b) shows (i) the relative differences of the 3D St values with respect to the 2D $S t$ value at $R e=168$, and (ii) the relative differences of the 3D $S t^{*}$ values with respect to the $2 \mathrm{D} S t^{*}$ value at $R e=168$.

At the onset of the secondary wake instability of $R e=165.7$, the relative difference between the 3D and 2D St values is approximately $6 \%$, while the relative difference between the normalised 3D and 2D $S t^{*}$ values reduces to less than $1 \%$. This suggests that the sudden drop of the 3D $S t$ from its 2D counterpart at $R e=165.7$ is due to the sudden decrease in the separating velocity (Fig. 9a) and the sudden increase in the wake width (Fig. 9b) at the onset of the secondary wake instability.

The $3 \mathrm{D} S t^{*}$ values are relatively uniform for $R e=165.7-210$, followed by an obvious increase starting at $R e$ of approximately 210 to 220 and a sharp decrease starting at $R e$ of approximately 320 to 330 (Fig. 10b). Compared with the original 3D $S t-R e$ relationship, after normalisation the $3 \mathrm{D} S t^{*}-$ Re relationship reveals better the two critical conditions of $R e \sim 210$ and 330 that have been identified in Fig. 3(a-e). 
However, the two critical conditions in Fig. 10(b) are less distinct than those shown in Fig. 3(a-e), since the separating velocity $U_{s}$ used in the normalisation of $S t$ is an approximation of the regional flow rate immediately prior to the rolling up of the vortices.

As an analogy to the case of a circular cylinder reported in Jiang and Cheng (2017), the increase in the 3D $S t^{*}$ as $R e$ exceeds 210 for a square cylinder is because the flow becomes increasing turbulent with increasing $R e$, and for turbulent flows the vortex shedding frequency no longer scales on $U_{s}$ and $D^{\prime}$. Specifically, the cross-flow distance for the vortex from one side of the cylinder to roll up to trigger the shedding of the vortex on the opposite side becomes increasingly smaller than $D^{\prime}$. In other words, the vortex shedding frequency scales on a length which is increasingly smaller than $D^{\prime}$. When $D^{\prime}$ is used to normalise the vortex shedding frequency, the 3D $S t^{*}$ value would be over-estimated.

Fig. 12 shows instantaneous $\omega_{z}$ contours of the span-averaged 3D flows for different $R e$ values, at the time instant that the streamwise velocity $u_{x}$ on the lower side of the cylinder is about to increase (i.e. $u_{x}$ is the smallest at this time instant) and the lower shear layer is about to roll up to form a new vortex (which is indicated by an arrow in Fig. 12). As Re increases from 210 to 330, vortex shedding occurs when the negative vortex is further away from the positive vortex being shed (Fig. 12a,b). As $R e$ increases from 330 to 400 , the opposite trend happens (Fig. 12b,c), such that the 3D $S t^{*}$ (which is normalised based on $D^{\prime}$ ) decreases. 
(a)

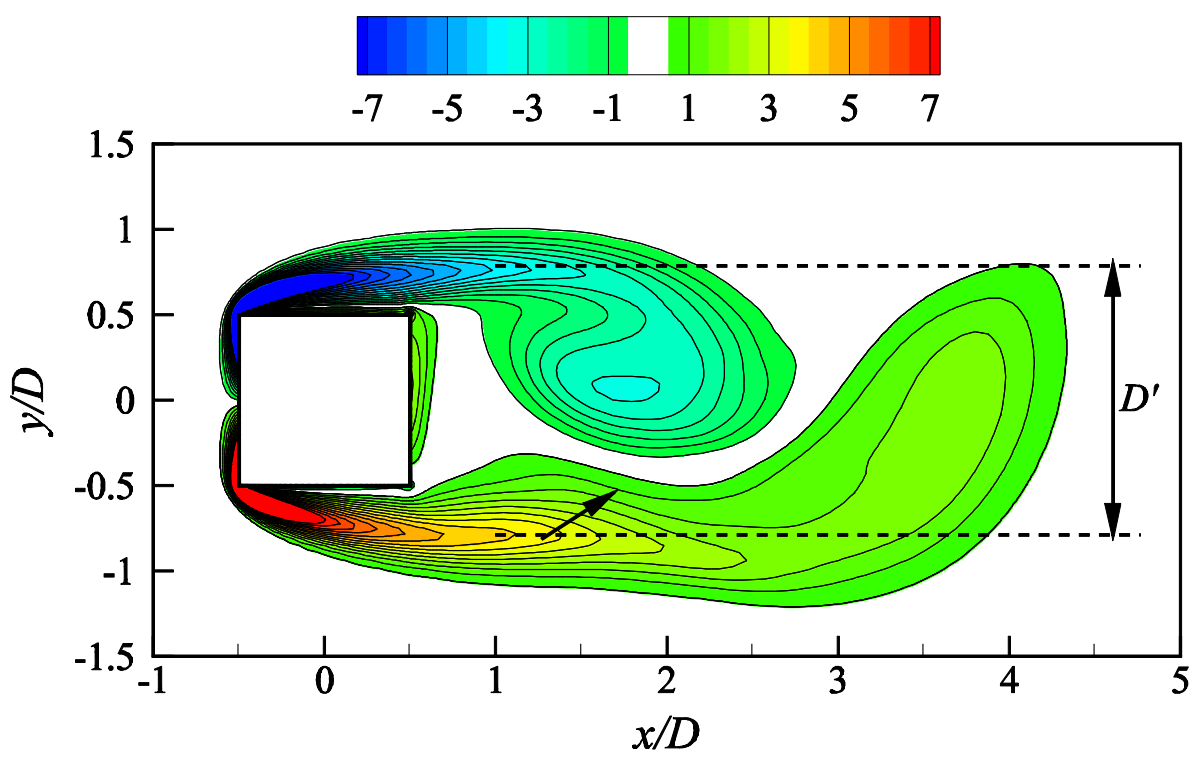

(b)

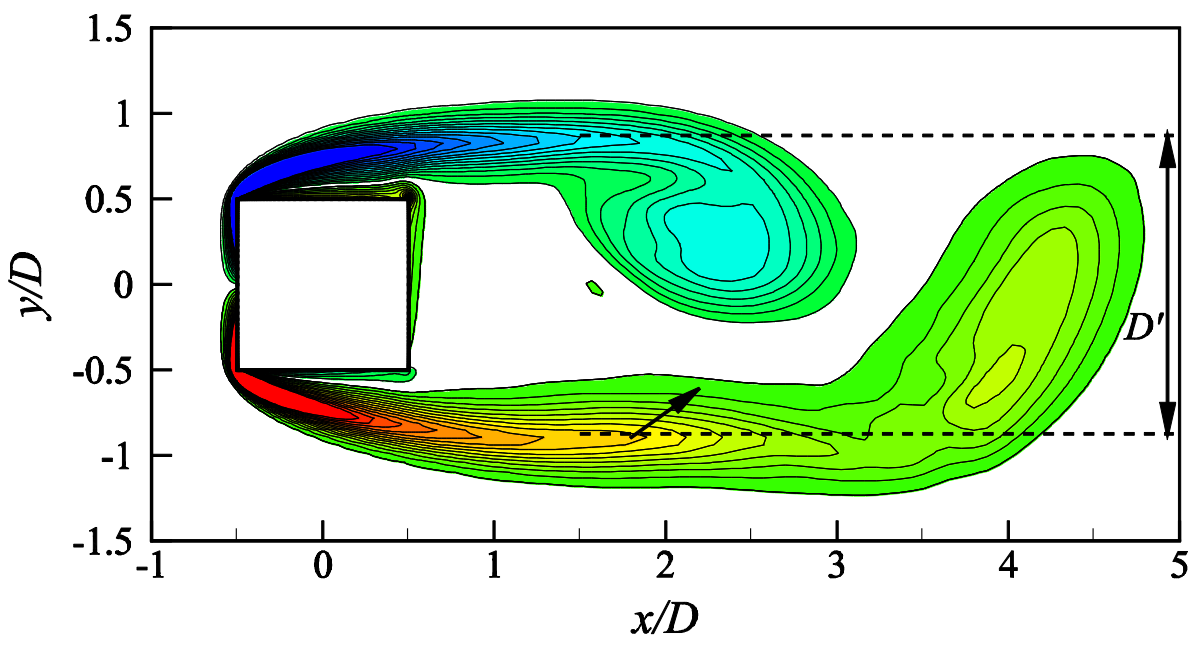

(c)

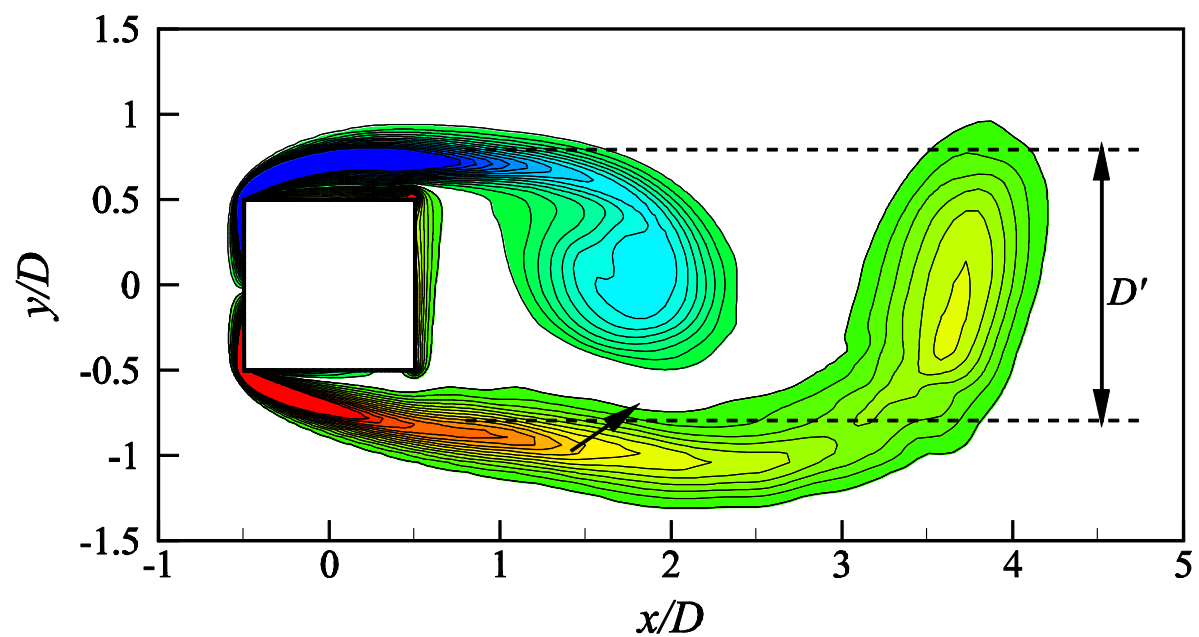

Fig. 12. Instantaneous $\omega_{z}$ contours of the span-averaged 3D flows for (a) $R e=210$, (b) $R e=330$, and (c) $R e=375$. The wake width is obtained from the $\omega_{z}$ contours of the time- and span-averaged flow. The arrow on the lower shear layer marks the location where the lower shear layer is about to roll up to form a new vortex. 


\subsection{Critical conditions for the 3D flows}

At the onset of the secondary wake instability of $R e=165.7$, a sudden increase or decrease of the $3 \mathrm{D}$ value with respect to its $2 \mathrm{D}$ counterpart is observed for each of the flow properties shown in Fig. 3 as well as the viscous and pressure drag coefficients shown in Fig. 6(a). This is due to the sudden increase in the flow three-dimensionality at the mode A instability that is subcritical in nature (Jiang et al., 2018).

For the 3D flows beyond the secondary wake instability, two critical conditions are observed at $R e$ of approximately 210 and 330. The critical condition at $R e \sim 210$ is due to the development of increasingly disordered mode B flow structures (i.e. increasingly turbulent wake flow) at $R e \geq 210$ (Fig. 5(c,d)), such that the deviations between the 3D and 2D hydrodynamic forces increase with increasing $R e$ values (Fig. 3(a-d)). The critical point of $R e \sim 210$ is slightly $(\Delta R e \sim 10)$ above the global instability of mode B (e.g. $R e=198$ in Sheard et al. (2009) and $R e=201$ in Choi et al. (2012), Park and Yang (2016) and Jiang et al. (2018)). We found that this conclusion is also valid for the case of flow past a circular cylinder, for which a similar critical condition is observed at $R e \sim 270$ (Williamson, 1996a), while the global instability of mode B occurs at $R e=259$ (Barkley and Henderson, 1996), again showing a difference of $\Delta R e \sim 10$.

The critical condition at $R e \sim 330$ is related to the disappearance and reappearance of the recirculation regions on the upper and lower sides of the cylinder. Fig. 13 plots the recirculation regions for a few $R e$ values obtained with the time- and span-averaged 3D flow. In addition, the evolution of the size of the recirculation region with $R e$ is indicated in Fig. 13 by the left and right ends of the recirculation region. It is noted that the right end is the saddle point illustrated in Fig. 11. As Re increases from 167 (the onset of flow reversal) to 330, the amount of flow travelling from the wake to the upper and lower sides of the cylinder increases monotonically (Fig. 14), such that the recirculation region is diverted away from the cylinder, and the size of the recirculation region is squeezed by the reverse flow from the lower, left 
and upper sides (see Fig. 13). At $R e=330$, the recirculation region almost disappears, which leads to a critical condition. Unlike the critical conditions at the onset of the secondary wake instability (of $R e=165.7$ ) and at the point where the wake flow becomes increasingly turbulent (of $R e \sim 210$ ), such a critical condition at $R e \sim 330$ for a square cylinder is not observed for a circular cylinder, since the geometry of a circular cylinder does not give rise to such a flow reversal.

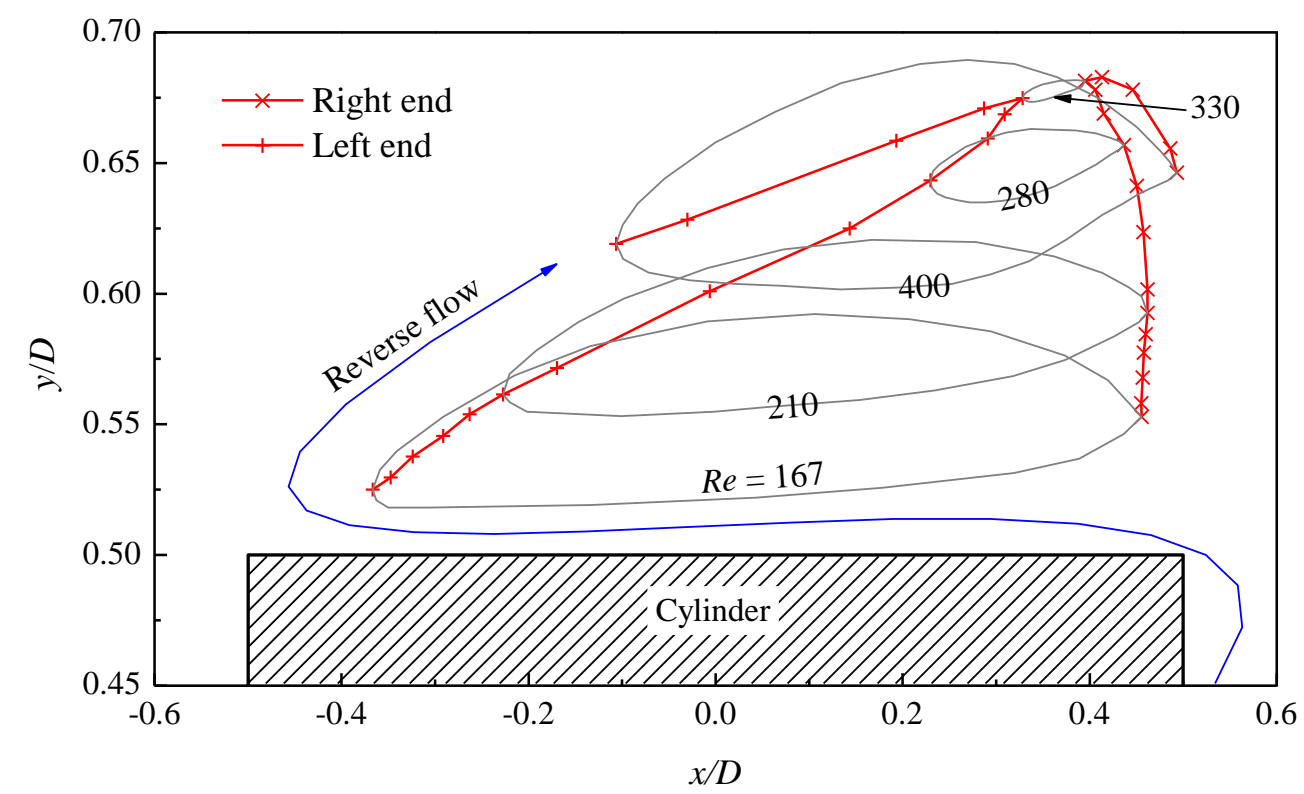

Fig. 13. The recirculation region on the upper side of the cylinder obtained with the time- and span-averaged 3D flow. Different horizontal and vertical scales are used to allow for a better visualization of the recirculation region. 


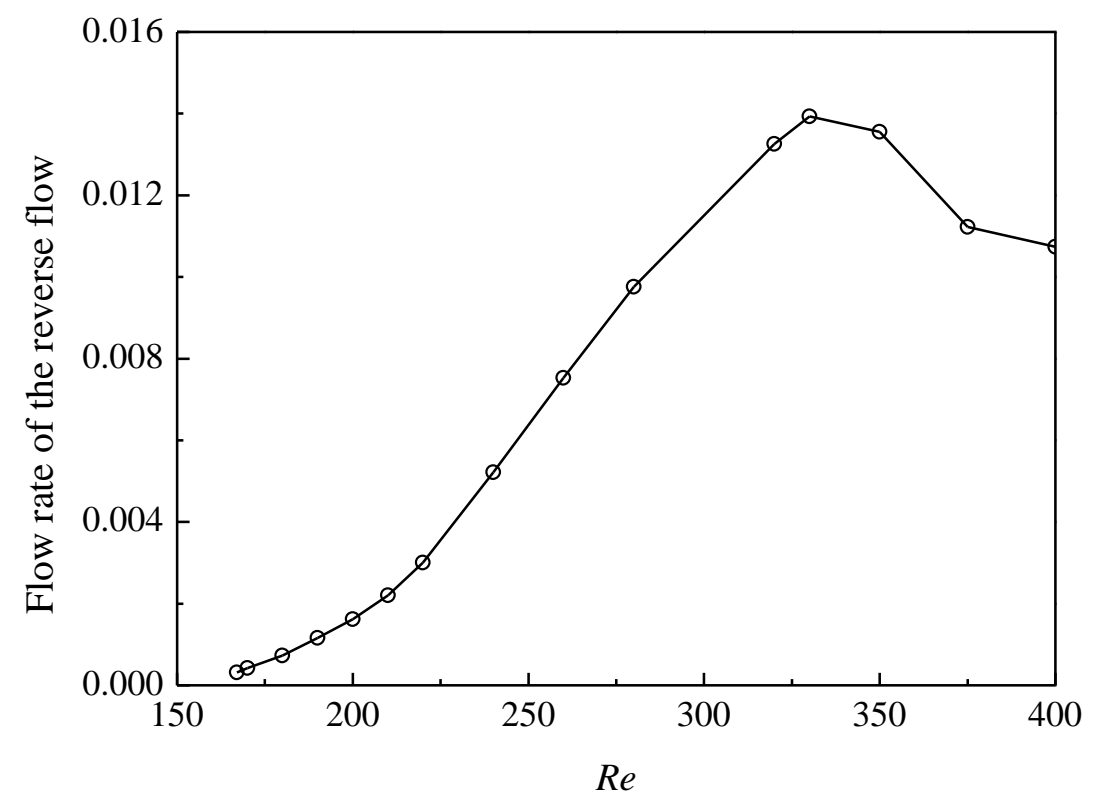

Fig. 14. Flow rate of the reverse flow in the time- and span-averaged 3D flow field. The flow rate is integrated vertically from the right end of the upper recirculation region (i.e. the saddle point) down to the cylinder surface.

\section{Conclusions}

This paper presents a DNS study of the 2D and 3D hydrodynamic forces and wake flow characteristics for flow past a square cylinder. The hydrodynamic forces and wake flow characteristics are presented for a range of $R e$ from 10 to 400, with a high resolution in $R e$ to capture all the variation trends.

Distinct features in the variation trends of the hydrodynamic forces with $R e$ are examined. A local minimum in the time-averaged drag coefficient is observed at $R e=$ 144 , which is due to combined contributions of the pressure drag and viscous drag. It is also found that the viscous drag decreases to negative values for $R e \geq 154$, which is due to the increasing coverage of backward flow on the upper and lower surfaces of the cylinder. For the 2D St-Re relationship, a local maximum is observed at $R e=168$. This is because the separating velocity is the largest and correspondingly the wake width is the smallest at $R e$ very close to 168 . The decrease in the separating velocity for $R e>168$ is due to the flow entrainment from the wake recirculation region to the upper and lower sides of the cylinder. For the 3D $S t-R e$ relationship, a sudden drop of 
the 3D St value from its 2D counterpart is observed at the onset of the secondary wake instability, which is due to the sudden decrease in the separating velocity and the sudden increase in the wake width at this point.

For the 3D flows, three critical conditions are observed at $R e$ of approximately 165.7, 210 and 330. At the onset of the secondary wake instability of $R e=165.7$, a sudden increase or decrease of the 3D flow property with respect to its $2 \mathrm{D}$ counterpart is observed, which is due to the sudden increase in the flow three-dimensionality at the mode A instability that is subcritical in nature. The second critical condition happens at $R e \sim 210$. As $R e$ exceeds 210 , the 3D flow properties are increasingly deviated from their 2D counterparts, which is because for $R e>210$ the mode B flow structure becomes increasingly disordered and the wake flow becomes increasingly turbulent. The critical point of $\operatorname{Re} \sim 210$ is slightly $(\Delta R e \sim 10)$ above the global instability of mode $\mathrm{B}$ of $R e \sim 201$. This conclusion is also applicable to a circular cylinder. The third critical condition occurs at $R e \sim 330$, which is related to the disappearance and reappearance of the recirculation regions on the upper and lower sides of the cylinder. This critical condition is not observed for the case of a circular cylinder, since the geometry of a circular cylinder does not give rise to recirculation regions on the upper and lower sides of the cylinder.

\section{Acknowledgements}

The authors would like to acknowledge the support from the Australian Research Council through LP150100249. This work was supported by resources provided by the Pawsey Supercomputing Centre with funding from the Australian Government and the Government of Western Australia.

\section{References}

Bai, H., Alam, M.M., 2018. Dependence of square cylinder wake on Reynolds number. Physics of Fluids 30, 015102.

Barkley, D., Henderson, R.D., 1996. Three-dimensional Floquet stability analysis of the wake of a 
circular cylinder. Journal of Fluid Mechanics 322, 215-241.

Choi, C., Jang, Y., Yang, K., 2012. Secondary instability in the near-wake past two tandem square cylinders. Physics of Fluids 24, 024102.

Henderson, R.D., 1995. Details of the drag curve near the onset of vortex shedding. Physics of Fluids 7, 2102.

Henderson, R.D., 1997. Nonlinear dynamics and pattern formation in turbulent wake transition. Journal of Fluid Mechanics 352, 65-112.

Issa, R.I., 1986. Solution of implicitly discretized fluid flow equations by operator-splitting. Journal of Computational Physics 62, 40-65.

Issa, R.I., Gosman, A.D., Watkins, A.P., 1986. The computation of compressible and incompressible recirculating flows by a non-iterative implicit scheme. Journal of Computational Physics 62, 66-86.

Jiang, H., Cheng, L., Draper, S., An, H., Tong, F., 2016. Three-dimensional direct numerical simulation of wake transitions of a circular cylinder. Journal of Fluid Mechanics 801, $353-391$.

Jiang, H., Cheng, L., 2017. Strouhal-Reynolds number relationship for flow past a circular cylinder. Journal of Fluid Mechanics 832, 170-188.

Jiang, H., Cheng, L., An, H., 2017. On numerical aspects of simulating flow past a circular cylinder. International Journal for Numerical Methods in Fluids, 85, 113-132.

Jiang, H., Cheng, L., An, H., 2018. Three-dimensional wake transition of a square cylinder. Journal of Fluid Mechanics 842, 102-127.

Luo, S.C., Chew, Y.T., Ng, Y.T., 2003. Characteristics of square cylinder wake transition flows. Physics of Fluids 15, 2549-2559.

Luo, S.C., Tong, X.H., Khoo, B.C., 2007. Transition phenomena in the wake of a square cylinder. Journal of Fluids and Structures 23, 227-248.

Moukalled, F., Mangani, L., Darwish, M., 2016. The finite volume method in computational fluid dynamics, Springer International Publishing, Switzerland.

Noca, F., Park, H., Gharib, M., 1998. Vortex formation length of a circular cylinder $(300<R e<$ 4000) using DPIV. Proceedings on Bluff Body Wakes and Vortex-Induced Vibration, ASME Fluids Engineering Division, Washington, DC. 
Norberg, C., 2003. Fluctuating lift on a circular cylinder: review and new measurements. Journal of Fluids and Structures 17, 57-96.

Okajima, A., 1982. Strouhal numbers of rectangular cylinders. Journal of Fluid Mechanics 123, $379-398$

Park, D., Yang, K., 2016. Flow instabilities in the wake of a rounded square cylinder. Journal of Fluid Mechanics 793, 915-932.

Pedersen, J.R., Larsen, B.E., Bredmose, H., Jasak, H., 2017. A new volume-of-fluid method in OpenFOAM. VII International Conference on Computational Methods in Marine Engineering, Nantes, France.

Posdziech, O., Grundmann, R., 2007. A systematic approach to the numerical calculation of fundamental quantities of the two-dimensional flow over a circular cylinder. Journal of Fluids and Structures 23, 479-499.

Robichaux, J., Balachandar, S., Vanka, S.P., 1999. Three-dimensional Floquet instability of the wake of square cylinder. Physics of Fluids 11, 560-578.

Roshko, A., 1955. On the wake and drag of bluff bodies. Journal of the Aeronautical Sciences 22, $124-132$.

Saha, A.K., Muralidhar, K., Biswas, G., 2000. Transition and chaos in two-dimensional flow past a square cylinder. Journal of Engineering Mechanics 126, 523-532.

Saha, A.K., Biswas, G., Muralidhar, K., 2003. Three-dimensional study of flow past a square cylinder at low Reynolds numbers. International Journal of Heat and Fluid Flow 24, 54-66.

Seng, S., Monroy, C., Malenica, Š., 2017. On the use of Euler and Crank-Nicolson time-stepping schemes for seakeeping simulations in OpenFOAM. VII International Conference on Computational Methods in Marine Engineering, Nantes, France.

Sheard, G.J., Fitzgerald, M.J., Ryan, K., 2009. Cylinders with square cross-section: wake instabilities with incidence angle variation. Journal of Fluid Mechanics 630, 43-69.

Sohankar, A., Norberg, C., Davidson, L., 1997. Numerical simulation of unsteady low-Reynolds number flow around rectangular cylinders at incidence. Journal of Wind Engineering and Industrial Aerodynamics 69-71, 189-201.

Sohankar, A., Norberg, C., Davidson, L., 1999. Simulation of three-dimensional flow around a square cylinder at moderate Reynolds numbers. Physics of Fluids 11, 288-306. 
Versteeg, H.K., Malalasekera, W., 1995. An introduction to computational fluid dynamics (second edition). Bell \& Bain Limited, Glasgow.

Vukčević, V., Jasak, H., Malenica, Š., 2016. Decomposition model for naval hydrodynamic applications, Part I: Computational method. Ocean Engineering 121, 37-46.

Williamson, C.H.K., 1989. Oblique and parallel modes of vortex shedding in the wake of a circular cylinder at low Reynolds numbers. Journal of Fluid Mechanics 206, 579-627.

Williamson, C.H.K., 1996a. Vortex dynamics in the cylinder wake. Annual Review of Fluid Mechanics 28, 477-539.

Williamson, C.H.K., 1996b. Three-dimensional wake transition. Journal of Fluid Mechanics 328, $345-407$.

Williamson, C.H.K., Brown, G.L., 1998. A series in $1 / \sqrt{ }$ Re to represent the Strouhal-Reynolds number relationship of the cylinder wake. Journal of Fluids and Structures 12, 1073-1085.

Williamson, C.H.K., Roshko, A., 1990. Measurements of base pressure in the wake of a cylinder at low Reynolds numbers. Zeitschrift fuer Flugwissenschaften und Weltraumforschung 14, $38-46$.

Wu, M.H., Wen, C.Y., Yen, R.H., Weng, M.C., Wang, A.B., 2004. Experimental and numerical study of the separation angle for flow around a circular cylinder at low Reynolds number. Journal of Fluid Mechanics 515, 233-260. 